\title{
Endothelial Adora2a Activation Promotes Blood-Brain Barrier Breakdown and Cognitive Impairment in Mice with Diet-Induced Insulin Resistance
}

\author{
Masaki Yamamoto, ${ }^{1} \oplus^{-D e-H u a n g ~ G u o,}{ }^{1}$ Caterina M. Hernandez, ${ }^{2}$ and ${ }^{\circledR}$ Alexis M. Stranahan ${ }^{1}$ \\ Departments of ${ }^{1}$ Neuroscience and Regenerative Medicine, and ${ }^{2}$ Pharmacology and Toxicology, Medical College of Georgia, Augusta University, Augusta, \\ Georgia 30912
}

Obesity and insulin resistance elicit blood- brain barrier (BBB) breakdown in humans and animal models, but the relative contributions of the two pathologies remain poorly understood. These studies initially addressed the temporal progression of cerebrovascular dysfunction relative to dietary obesity or diet-induced insulin resistance in male mice. Obesity increased BBB permeability to the low molecular weight fluorophore sodium fluorescein $(\mathrm{NaFl})$, whereas diet-induced insulin resistance increased permeability to both $\mathrm{NaFl}$ and Evans blue, which forms a high molecular weight complex with serum albumin. Serial section transmission electron microscopy analysis of hippocampal capillaries revealed that diabetes promotes involution of tight junctions, fenestration of endothelial cells, and pericyte regression. Chronic activation of adenosine receptor 2a (Adora2a) erodes tight junctions between endothelial cells of the cerebral vasculature in other models of chronic neuropathology, and we observed that acute Adora2a antagonism normalized BBB permeability in wild-type mice with diet-induced insulin resistance. Experiments in mice with inducible deletion of Adora2a in endothelial cells revealed protection against BBB breakdown with diet-induced insulin resistance, despite comparable metabolic dysfunction relative to nontransgenic littermates. Protection against BBB breakdown was associated with decreased vascular inflammation, recovery of hippocampal synaptic plasticity, and restoration of hippocampus-dependent memory. These findings indicate that Adora2a-mediated signaling in vascular endothelial cells disrupts the BBB in dietary obesity, and implicate cerebrovascular dysfunction as the underlying mechanism for deficits in synaptic plasticity and cognition with obesity and insulin resistance.

Key words: blood- brain barrier; diabetes; hippocampus; neurovascular; obesity

Significance Statement

The blood- brain barrier (BBB) restricts the entry of circulating factors into the brain, but obesity promotes BBB breakdown in humans and animal models. We used transgenic mice with resistance to BBB breakdown to investigate the role of neurovascular dysfunction in high-fat diet (HFD)-induced cognitive impairment. Transgenic mice with inducible ablation of Adora2a in endothelial cells were protected against BBB breakdown on HFD, despite comparable metabolic impairments relative to normal mice. Transgenic mice were also resistant to HFD-induced cognitive dysfunction and were protected against deficits in hippocampal synaptic plasticity. These findings indicate that Adora2a-mediated signaling in endothelial cells mediates obesity-induced BBB breakdown, and implicate cerebrovascular dysfunction as the mechanism for deficits in synaptic plasticity and cognition with obesity and diabetes.

\section{Introduction}

Obesity and diabetes are increasingly recognized as risk factors for age-related cognitive decline (Biessels and Reagan, 2015). In-

\footnotetext{
Received Sept. 28, 2018; revised Feb. 18, 2019; accepted March 6, 2019.

Author contributions: M.Y., D.-H.G., C.M.H., and A.M.S. designed research; M.Y., D.-H.G., C.M.H., and A.M.S. performed research; M.Y., D.-H.G., C.M.H., and A.M.S. analyzed data; M.Y. wrote the first draft of the paper; M.Y., D.-H.G., C.M.H., and A.M.S. edited the paper; A.M.S. contributed unpublished reagents/analytic tools; A.M.S. wrote the paper.

This work was supported by Grants R01DK110586 and K01DK100616 from the NIH to A.M.S. Behavioral testing was performed with assistance from the Small Animal Behavior Core at Augusta University. Electron microscopy experiments were carried out assistance from the Electron Microscopy Core Facility at Augusta University.
}

dividuals with type 2 diabetes and obesity exhibit higher rates of cognitive impairment with aging in cross-sectional and longitudinal studies (Elias et al., 2005; Whitmer et al., 2008; Xu et al., 2010). Changes in brain structure with obesity and diabetes are prominent in the hippocampus, which mediates memory forma-

\footnotetext{
The authors declare no competing financial interests.
}

Correspondence should be addressed to Alexis M. Stranahan at astranahan@augusta.edu.

C. M. Hernandez' present address: Department of Pharmaceutical Sciences, Appalachian College of Pharmacy, Oakwood, VA 24631.

https://doi.org/10.1523/JNEUROSCI.2506-18.2019

Copyright $\odot 2019$ the authors 
tion and exhibits early vulnerability to aging and Alzheimer's disease (AD; Ward et al., 2005; Ho et al., 2010). Adipose tissue hypertrophy and ectopic lipid deposition both contribute to chronic inflammation in obese individuals, and emerging evidence suggests that attenuating inflammation in the brain mitigates the neurocognitive consequences of obesity (Kanneganti and Dixit, 2012; Erion et al., 2014). Although the blood-brain barrier (BBB) was classically thought to prevent systemic inflammation from reaching the brain, obesity and aging compromise BBB integrity in humans and rodent models (Gustafson et al., 2007; Kanoski et al., 2010; Montagne et al., 2015; Stranahan et al., 2016). This shared feature could allow peripheral inflammation to impair neuronal function and might contribute to the comorbidity between obesity and cognitive decline.

Circulating factors have a restricted set of routes through the intact BBB. Cerebrovascular endothelial cells express saturable transporters for hormones and cytokines, including inflammatory cytokines and metabolic hormones (Banks, 2015). Steroid hormones and other lipid-soluble circulating factors pass more readily through the $\mathrm{BBB}$, either via the transcellular lipophilic pathway, or with assistance from P-glycoprotein and other efflux transporters (Abbott et al., 2006). Under normal conditions, paracellular permeability is limited by tight junctions between adjacent endothelial cells, which are formed during ontogeny of the cerebrovasculature (Daneman and Prat, 2015). Cerebrovascular tight junctions comprised of claudin proteins and occludin anchor the adjacent endothelial cell membranes to one another, forming the structural basis of the BBB (Bauer et al., 2014; Zhao et al., 2015). Reductions in tight junction proteins have been reported in rodent models of obesity and diabetes (Ouyang et al., 2014; Stranahan et al., 2016), but the relative contributions of obesity and diabetes have yet to be elucidated.

Endothelial cells and pericytes make up the luminal side of the $\mathrm{BBB}$, and astroglial endfeet comprise the abluminal component (Abbot et al., 2006). Reactive astrogliosis has been reported in rodent models of obesity (Buckman et al., 2013; Calvo-Ochoa et al., 2014), but the source and identity of signals driving astrogliosis has yet to be determined. Angiogenesis is a recognized feature of diabetic retinopathy and pathological neovascularization has been reported in the hypothalamic arcuate nucleus with obesity (Yi et al., 2012). However, the adverse neuronal effects of dietinduced insulin resistance are unlikely to be a direct consequence of increased vascular innervation, as angiogenesis is associated with the beneficial effects of flavonols and environmental enrichment on neuroplasticity (Isaacs et al., 1992; van Praag et al., 2007). The opposing consequences of flavonol- and enrichmentinduced angiogenesis for neuronal synapses, relative to the synaptopathic effects of neovascularization in diabetes, implies that features of the local environment may determine the synaptic consequences of new vessel formation. However, the signaling mechanisms linking vascular function with neuroplasticity are still being elucidated.

These studies were initiated with the goal of determining whether changes in vascular permeability occurred as a function or obesity or diabetes. After identifying the critical windows for obesity-induced cerebrovascular disruption, relative to the onset of metabolic and cognitive dysfunction, we generated a novel transgenic mouse line with inducible resistance to obesity-induced cerebrovascular dysfunction. Adenosine receptor 2a (Adora2a) was ablated in Tie2-expressing endothelial cells using a doxycyclineinducible rtTA to drive cre recombinase (AR2ATg), and transgenic mice were maintained on either high-fat diet (HFD) or low-fat diet (LFD) before analysis of metabolic, neurovascular, and cognitive endpoints. These studies suggest that changes in cerebrovascular permeability initiate the cycle of obesity-induced neuroinflammation and cognitive impairment.

\section{Materials and Methods}

Animals, experimental diets, and drug administration. For initial time course experiments, male C57BL6J mice were purchased from Jackson Laboratories or bred in-house before being exposed to increasing durations of HFD (60\% fat; Research Diets, D12492) or LFD (10\% fat; Research Diets, D12450). After characterizing the temporal progression of cerebrovascular dysfunction relative to obesity and insulin resistance, additional groups of wild-type (Wt) mice were maintained on HFD or LFD for 16 weeks before administration of vehicle or the selective Adora2a antagonist SCH58261 $(0.1 \mathrm{mg} / \mathrm{kg}$ in saline containing $1 \%$ Tween 80, i.p., every $12 \mathrm{~h}$ for $3 \mathrm{~d}$; Tocris Bioscience). To generate transgenic mice with inducible ablation of Adora2a in endothelial cells, male Tie2 ${ }^{\text {rtTA }}$ founders (Jax strain ID 005493) were bred with Adora2 $\mathrm{a}^{\mathrm{fl} / \mathrm{fl}}$ females (donated by Joel Linden, Salk Institute; available at Jackson Laboratories, strain ID 010687) to generate Tie $2^{\mathrm{rtTA}} /$ Adora2 $\mathrm{a}^{\mathrm{fl} / \mathrm{fl}}$ mice. Male Tie ${ }^{\text {rtTA}} /$ Adora2a ${ }^{\mathrm{fl} / \mathrm{fl}}$ mice were then crossed with female tetO ${ }^{\text {cre }}$ mice (Jax strain ID 006234) to generate mice with doxycycline-inducible ablation of Adora2a in endothelial cells. Female Tie $2^{\text {rtTA }}$ mice were not used due to germline transmission of the flox/ $\Delta$ allele in females (Koni et al., 2001).

Male Tie $2^{\text {rtTA }} /$ tetO $^{\text {cre }} /$ Adora $2 \mathrm{a}^{\text {fl/fl }}$ mice and nontransgenic male littermates were maintained on HFD for 16 weeks as detailed above, with doxycycline administration during Week 12 ( $2 \mathrm{mg} / \mathrm{d}$ for $7 \mathrm{~d}$, PO). Body weights and food intake were measured weekly throughout each experiment and intraperitoneal glucose tolerance tests were performed during Week 14, as described previously (Dey et al., 2014). Mice were genotyped using a commercial genotyping service (Transnetyx) with randomly selected tails from each litter validated using the recommended primer sequences from Jackson Laboratories. All experiments followed NIH guidelines and were approved by the Animal Care and Use Committee at the Medical College of Georgia.

Behavioral testing. Testing in the novel object preference paradigm was performed during the first half of the dark phase, as described previously (Wosiski-Kuhn et al., 2014). In brief, for novel object recognition testing, mice were exposed to two identical objects for $10 \mathrm{~min}$, then returned to the home cage for $30 \mathrm{~min}$ before the first test session with one novel and one familiar object. After the first test session, mice were returned to the home cage for $90 \mathrm{~min}$ before the second test session with one novel and one familiar object. The third test session was performed the next day at lights-out $(18: 00 \mathrm{~h})$ and all test sessions were $5 \mathrm{~min}$ in length. The arena was cleaned with $2 \%$ Bac-Down (Decon Labs) between trials. For Y-maze testing, we used a modified version of previously published protocols (Erion et al., 2014; Wosiski-Kuhn et al., 2014). Modifications involved analysis during spontaneous alternation, rather than the forced-choice paradigm used previously. Testing in the water maze also followed previously published methods (Stranahan et al., 2010), with minor modifications involving the use of a $5 \mathrm{ft}$ diameter pool. In brief, mice received four $90 \mathrm{~s}$ acquisition trials with a $10 \mathrm{~min}$ intertrial interval. Start points were varied pseudorandomly over $5 \mathrm{~d}$ of hidden platform training. For the probe trial, mice were placed into the water in the opposite quadrant and allowed to swim for $90 \mathrm{~s}$. Visible platform testing consisted of four $90 \mathrm{~s}$ trials, with start points at each corner of the pool. Distances, latencies, and swim speeds were determined using Anymaze software (Stoelting).

Analysis of BBB permeability. BBB permeability was determined using combined injections of sodium fluorescein $(\mathrm{NaFl} ; 10 \mathrm{mg}$ in $0.1 \mathrm{ml}$, i.p.; Miner et al., 2015) and Evans blue (50 mg in $0.1 \mathrm{ml}$, i.p.; Wang et al., 2004), using protocols modified from previously reported studies (Stranahan et al., 2016). In brief, cardiac blood was collected immediately before transcardial perfusion with saline $45 \mathrm{~min}$ after tracer injections. Hippocampal tissue and plasma samples were snap frozen on dry ice and stored at $-80 \mathrm{C}$ in preparation for trichloroacetic acid (TCA) extraction according to published methodology (Stranahan et al., 2016). NaFl was quantified at $480 \mathrm{~nm}$ excitation/520 $\mathrm{nm}$ emission on a Biotek plate reader 
and concentrations were determined using a standard curve containing known concentrations of NaFl diluted in TCA-clarified hippocampal tissue from an uninjected mouse. Evans blue was quantified in TCAclarified lysates diluted 1:4 in absolute ethanol, with readings at $620 \mathrm{~nm}$ excitation/680 nm emission, as described previously (Wang and Lai, 2014). Serum samples were quantified in parallel with clarified lysates for each tracer.

Brain cell isolation and flow cytometry. Isolation of microvessels (MVs) and brain vascular endothelial cells (BVECs) was performed as described previously (Wu et al., 2003), with modifications. Specifically, for microvessel isolation, mouse forebrains were bisected and the midline white-matter tracts were removed and discarded. Brains were homogenized in vascular isolation buffer (VIB) containing 10\% Accumax and cells were pelleted by centrifugation at $720 \times g$ for $5 \mathrm{~min}$. Cell pellets were resuspended in $5 \mathrm{ml}$ VIB and layered over 15\% dextran (Sigma-Aldrich). Gradients were separated by centrifugation at $1200 \times g$ for $1 \mathrm{~h}$ and the resultant pellet containing MVs was washed and digested in $0.5 \%$ collagenase before being passed through a $100 \mu \mathrm{m}$ cell strainer. The flowthrough was subsequently applied to a $40 \mu \mathrm{m}$ cell strainer and MVs were collected and pelleted by centrifugation before being frozen for flow cytometry and qPCR endpoints. For flow cytometry, MVs were labeled with $\mathrm{mAb}$ anti-CD45 (AbD, Serotec) and mAb anti-CD31 (BD Biosciences) after blocking Fc receptors and determination of cell viability with Viacount staining. Endothelial cells were gated into the SSClow/FSCmid/ CD45-/CD31+ population and e-selectin or ICAM-1 (BD Pharmingen) labeling was expressed relative to total gated events. In some experiments, forebrain mononuclear cells (FMCs) and astrocytes were isolated according to previously published methods (Stranahan et al., 2016), with modifications involving separation along a $75 / 50 / 30 \%$ gradient of isotonic Percoll. Astrocytes were collected from the 50/30\% interphase and FMCs were collected from the 75/50\% interphase. Cell pellets were snap frozen and stored at -80 in preparation for DNA extraction, as described previously (Stranahan et al., 2011).

Quantitative real-time PCR. For qPCR, total RNA was extracted from forebrain microvessel preparations according to published methods (Wosiski-Kuhn et al., 2014). After column purification (Qiagen), cDNA was synthesized from purified RNA template using a commercial kit (Applied Biosystems). For qPCR, $500 \mathrm{ng}$ cDNA was amplified using Applied Biosystems $2 \times$ MasterMix and TaqMan probes on a StepOne plus instrument. Expression was determined by calculating the ddCT, with average dCT for nontransgenic (nTg)/LFD samples as the reference group. The following TaqMan probes were used in these experiments: Adora1, Mm01308023_m1; Adora2a, Mm00802075_m1; Cldn1, Mm00516701_m1; Flt1, Mm00438980_m1; Glut1, Mm00441480_m1; Jam2, Mm00470197_m1; Kdr, Mm01222421_m1; Vegfa, Mm00437306_ ml; Vegfb, Mm00442102_ml; Gapdh, Mm99999915_g1.

For some experiments, genomic DNA was isolated from the phenol layer after collecting the aqueous phase for RNA extraction with the aid of Phasemaker separation tubes (Invitrogen). Genomic DNA from hippocampus and primary cells was amplified using primers targeted to the deleted sequence for Adora2a (F:TCACATCTCAAGTGTGGTGC; R:GCGGGTCTAGACGACTGTAA). Amplification products generated using standard PCR were separated by gel electrophoresis and imaged on a Li-Cor Odyssey imaging system.

Western blotting and ELISA. For Western blotting, protein extraction and separation by gel electrophoresis followed previously published methods (Erion et al., 2014). Immunodetection was performed using the following primary antibodies: rabbit anti-Claudin 5 (Abcam), rabbit anti-Occludin (Abcam), and mouse anti- $\beta$ actin (Sigma-Aldrich). Signals were detected using NIR-conjugated secondary antibodies (Li-Cor) and visualized on a Li-Cor Odessey imaging system. ELISA was performed on serum samples using commercially available kits according to the manufacturer's instructions (insulin, Crystal Chem; VEGF, R\&D Systems).

Hippocampal slice physiology and DiI labeling. Brain slice preparation and electrophysiological recording was performed as described previously (Hao et al., 2016). In brief, transverse hippocampal slices (350 $\mu \mathrm{m}$ thickness) were prepared after decapitation under Isoflurane anesthetic. After $1 \mathrm{~h}$ recovery at $37 \mathrm{C}$ in carboxygenated artificial CSF (ACSF), slices were transferred to a submerged recording chamber and electrodes were positioned in the middle molecular layer of the dentate gyrus. The medial perforant path was functionally identified by the presence of pairedpulse depression and stimulus intensity was set at $50 \%$ maximal based on the input/output curve. Baseline and poststimulation recordings were performed at $0.05 \mathrm{~Hz}$ and LTP was induced with a $1 \mathrm{~s}$ train delivered at $100 \mathrm{~Hz}$. Data were acquired and analyzed using pClamp v11 (Molecular Devices). All recordings were made in ACSF containing $50 \mu \mathrm{M}$ picrotoxin (Sigma-Aldrich) and a subset of recordings was performed in the presence of 50 nm SCH58261 (Tocris Bioscience).

To label dendritic spines, we used a modified juxtacellular DiI labeling protocol based on previously published methods (Hao et al., 2016). For this approach, glass micropipettes $(1.1 \mathrm{~mm}$, i.d.) were pulled to generate a tapered tip (0.1 mm diameter) using a Flaming-Brown micropipette puller (Sutter Instruments). DiI crystals (Invitrogen, catalog \#D282) were aspirated into the micropipette, which was positioned in the GCL of a fixed hippocampal slice (300 $\mu \mathrm{m}$ thickness) using a micromanipulator and dissecting scope. DiI crystals were loaded into the slice by pressurizing the micropipette through attached tubing and slices were incubated for $48-72 \mathrm{~h}$ at $37^{\circ} \mathrm{C}$ before visualization of spines on a Zeiss $780 \mathrm{mul}-$ tiphoton microscope. Quantification of dendritic spines was performed using Reconstruct freeware (https://synapseweb.clm.utexas.edu/), as described previously (Hao et al., 2016).

Immunofluorescence staining and image analysis. Tissue preparation for immunofluorescence staining followed previously published methods (Stranahan et al., 2016). Primary antibodies and reagents included biotinylated or Texas Red-conjugated tomato lectin (1:500; Vector Laboratories), rabbit anti-GFAP (1:1000; Pierce), rabbit anti-PDGFRbeta (1:500; Millipore; Sigma), rabbit anti-COX-2 (1:200; Santa Cruz Biotechnology), and rabbit anti-CD31 (1:200; BD Pharmingen). In brief, coronal sections were collected in a 1:6 series through the hippocampus using a freezing microtome. For lectin staining with streptavidin detection, sections were quenched in $0.3 \% \mathrm{H}_{2} \mathrm{O}_{2}$ diluted in the manufacturer's recommended buffer before incubation with biotinylated tomato lectin. For all other antigens, sections were quenched in $0.1 \% \mathrm{NaBH} 4$ diluted in Tris-buffered saline before being washed and incubated with the indicated concentration of primary antibody. After overnight incubation, sections were washed and blocked in normal sera before being incubated with secondary antibodies conjugated to AlexaFluor dyes (Invitrogen). To detect extravascular IgG deposits, sections were processed for lectin staining with Texas Red-conjugated tomato lectin followed by incubation with AlexaFluor 488-conjugated goat anti-mouse IgG (1:100; Invitrogen). After each reaction, sections were mounted on Superfrost Plus slides, dried, and counterstained with DAPI before being imaged on a Zeiss 780 upright confocal microscope.

For analysis of vascular area and tortuosity, image stacks were acquired at $25 \times$ magnification from the dentate gyrus molecular layer on $(8-10)$ serial sections (120 $\mu \mathrm{m}$ spacing). Image stacks were thresholded using the Otsu algorithm and segmented using the 3D skeletonize plugin in Fiji (https://imagej.net/AnalyzeSkeleton). Pericyte coverage of lectinpositive vascular profiles was determined by analysis of the area occupied by PDGFRb labeling relative to total vascular area on $z$-stack images. For quantification of extravascular IgG deposits, $z$-stack images of Texas Red-conjugated tomato lectin and mouse IgG were acquired as described above, and area occupied by lectin-labeled vascular profiles was subtracted from the area occupied by IgG immunoreactivity using the Image Calculator function in Fiji (https://imagej.nih.gov/ij/docs/menus/ process.html\#calculator). After excluding the area occupied by vasculature from the image stacks, the integrated density of extravascular IgG immunoreactivity was determined from $2 \mathrm{D}$ maximum intensity projection images (10 $\mu \mathrm{m}$ stack height). For clarity of presentation, the Texas Red-conjugated lectin signal was pseudocolored green and the IgG signal was pseudocolored red in manuscript figures. In some experiments, perivascular IgG deposits were analyzed in vascular microdomains with or without pericytes. For these experiments, lectin labeling was first used to delineate vascular regions-of-interest (ROIs) on maximum intensity projections, as described above. Within the vascular ROIs, PDGFRblabeled cells were used to delineate secondary ROIs for analysis of IgG deposits proximal and distal to pericytes. 
For analysis of perivascular GFAP immunoreactivity, multichannel image stacks of tomato lectin and GFAP were acquired from the dentate molecular layer. Total GFAP immunoreactivity was determined by calculating the area of suprathreshold labeling on $(8-10)$ sampled fields spanning the anteroposterior axis of the hippocampus. For each stack, tomato lectin-labeled vessels were used to delineate ROIs for analysis of perivascular GFAP labeling using the ROI manager plugin in ImageJ (https://imagej.nih.gov). The area of GFAP labeling within the tomato lectin ROIs was expressed relative to total GFAP labeling for statistical comparisons. All analyses were performed blind to treatment condition.

Serial section transmission electron microscopy. For serial section transmission electron microscopy (ssTEM), methods for transcardial perfusion and tissue fixation were consistent with previously published protocols (Wosiski-Kuhn et al., 2014). After 12-16 h postfixation, 100$\mu \mathrm{m}$-thick slices were cut on a Vibratome (Leica) and a $1.0 \mathrm{~mm}$ trapezoid was dissected from the middle molecular layer dorsal to the superficial blade of the dentate gyrus under a dissecting microscope. Trapezoids were postfixed in $2 \%$ osmium tetroxide in sodium cacodylate buffer, stained with $2 \%$ uranyl acetate in $70 \%$ ethanol, dehydrated in a graded ethanol series diluted in propylene oxide, and embedded in EponAraldite resin before being cured at $60^{\circ} \mathrm{C}$. Ultrathin sections were cut at $60 \mathrm{~nm}$ nominal thickness with a diamond knife on a Leica EM UC6 ultramicrotome (Leica Microsystems), collected on Pioloform-coated Synaptec copper slot grids (Electron Microscopy Sciences), and poststained with uranyl acetate and lead citrate for contrast enhancement. Images were acquired at $6000-8000 \times$ magnification on a JEM 1230 transmission electron microscope (JEOL; beam intensity $=110 \mathrm{kV}$ ) equipped with an UltraScan 4000 CCD camera and First Light Digital Camera Controller (Gatan).

Alignment and segmentation of TEM images was performed using Reconstruct software, as described previously (Wosiski-Kuhn et al., 2014). Only capillaries that traversed through $>150$ serial sections without bifurcating were used in these analyses. The areas of the endothelial basement membrane, endothelial cell bodies, tight junctions, and lumen were determined by manual segmentation of serial sections from $(n=3)$ mice on LFD or HFD for 16 weeks (mean \pm SEM $=205 \pm 10.1$ serial sections per vessel, 1 vessel per mouse). The $3 \mathrm{D}$ datasets were generated in parallel with $2 \mathrm{D}$ measures from single-section images of $(4-8)$ vessels per mouse. For combined analysis of the $2 \mathrm{D}$ and $3 \mathrm{D}$ datasets, area measurements from single-section micrographs were grouped with perseries average area measurements from serial images. The total volume of each capillary, including the lumen, was determined by tracing the outer basement membrane on each section to generate area measurements in square nanometers. The sum of all area measurements was converted to volume by multiplying by section thickness according to Cavalieri's principle (Cavalieri, 1635).

The area of the endothelial basement membrane was determined by tracing the inner basement membrane, then subtracting the area of the inner basement membrane from the area of the outer basement membrane on a per-section basis. The resultant area measurements were converted to volumes according to Cavalieri's principle as described above. To control for variability in the size of cells within the confines of the inner basement membrane, basement membrane volumes were normalized to the sum of endothelial cell volumes and pericyte volumes on the corresponding section. Tight junction volumes were determined by manual segmentation of tight junction areas on each section. The sum of all tight junction areas was converted to volume as described above and expressed relative to total endothelial cell volume for analysis.

Pericytes were distinguished from endothelial cells based on their separation from both the luminal and abluminal compartment by the pericyte basement membrane, as described previously (Yao et al., 2014). Pericyte volumes were determined by manual segmentation and expressed relative to total endothelial cell volumes on the corresponding section. Astroglial endfeet were identified by their cytoplasmic clarity, glycogen granules, occasional gliofilaments, and direct contact with the abluminal surface of the endothelial basement membrane. For analysis of endfoot swelling, astroglial processes with direct apposition to the endothelial basement membrane were traced in their entirety and the area of endfoot processes was expressed relative to capillary on the correspond- ing section. All tracings were conducted blind to experimental condition and verified by two independent experimenters.

Statistics. For the time-course experiments, neurovascular and physiological parameters were analyzed using two-way ANOVA with diet and time point as fixed factors. Significant differences at individual time points were determined using Bonferroni-corrected $t$ tests. For the ssTEM experiments, the effect of diet was determined using $t$ tests. When group differences in variance were present, the effect of diet was determined using Mann-Whitney's $U$ test. For the antagonist experiments and single-endpoint experimental measures in transgenic mice, data were analyzed using two-way ANOVA (diet $\times$ genotype) followed by post hoc Bonferroni-corrected $t$ tests as detailed above. Statistical analyses described in the preceding section were performed in GraphPad Prism v5 (GraphPad Software). Path lengths in the water maze during acquisition training and novel object preference data were analyzed using $2 \times 2$ repeated-measures ANOVA followed by Dunnett's post hoc (all comparisons relative to $\mathrm{nTg} / \mathrm{LFD}$ ). The aforementioned statistical comparisons were made using SPSS Statistics v25 (IBM Analytics). The threshold for all statistical comparisons was $p<0.05$.

\section{Results}

Time course for obesity-induced cerebrovascular dysfunction To determine whether changes in BBB permeability were attributable to obesity or diet-induced insulin resistance, male C57BL6J mice were maintained on HFD or LFD beginning at 8 weeks old. After 8, 12, or 16 weeks on either diet, mice were injected with $\mathrm{NaFl}$ and Evans blue and killed by transcardial perfusion with saline. HFD increased body weights at all time points examined (Fig. $1 A ; F_{(2,42)}=7.34, p<0.01$ ). Increases in fasting glucose and insulin concentrations were observed after 16 weeks HFD (Fig. $1 B$; for glucose, $F_{(2,42)}=5.82, p<0.05$; for insulin, $\left.F_{(2,42)}=6.06, p<0.05\right)$. Quantification of peripherally injected fluorophores in clarified hippocampal lysates revealed significant increases in vascular permeability in HFD mice (Fig. 1C). Increases in hippocampal $\mathrm{NaFl}$ were detected after 12 weeks HFD, before the development of fasting hyperglycemia $\left(F_{(2,42)}=4.10\right.$, $p<0.05)$. Evans blue extravasation was significantly increased after 16 weeks HFD (Fig. $1 C ; F_{(2,42)}=6.64, p<0.01$ ), after the development of hyperglycemia and hyperinsulinemia. Levels of $\mathrm{NaFl}$ and Evans blue in serum samples were unaffected by diet or time $\left(\mathrm{NaFl}, \mathrm{mg} / \mathrm{ml}\right.$, mean \pm SEM: $\mathrm{LFD}_{8 \mathrm{WK}}=8.7 \pm 0.41$, $\mathrm{HFD}_{8 \mathrm{WK}}=9.1 \pm 0.46, \mathrm{LFD}_{12 \mathrm{WK}}=8.5 \pm 0.53, \mathrm{HFD}_{12 \mathrm{WK}}=8.9 \pm$ $0.55, \mathrm{LFD}_{16 \mathrm{WK}}=8.63 \pm 0.61, \mathrm{HFD}_{16 \mathrm{WK}}=8.48 \pm 0.73$; Evans blue, $\mathrm{mg} / \mathrm{ml}$, mean $\pm \mathrm{SEM}: \mathrm{LFD}_{8 \mathrm{WK}}=42.6 \pm 1.24, \mathrm{HFD}_{8 \mathrm{WK}}=$ $40.3 \pm 0.85, \mathrm{LFD}_{12 \mathrm{WK}}=40.6 \pm 1.03, \mathrm{HFD}_{12 \mathrm{WK}}=41.4 \pm 1.10$, $\left.\mathrm{LFD}_{16 \mathrm{WK}}=40.5 \pm 0.6, \mathrm{HFD}_{16 \mathrm{WK}}=41.0 \pm 1.21\right)$. Analysis of extravascular IgG immunoreactivity in the dentate molecular layer revealed significant increases after 12 or 16 weeks HFD (Fig. $\left.1 D ; F_{(1,18)}=6.35, p<0.05\right)$, consistent with progressive erosion of the BBB with increasing durations of HFD consumption. This interpretation was also supported by quantification of the tight junction proteins claudin-5 and occludin in hippocampal lysates, which revealed reduced expression after 16 weeks HFD (Fig. 1E; for claudin-5: $F_{(2,36)}=9.79, p<0.01$; for occludin: $F_{(2,36)}=6.18$, $p<0.05)$.

Pathological angiogenesis is a hallmark of diabetic retinopathy and has also been demonstrated in the hypothalamic arcuate nucleus in rodents with dietary obesity (Yi et al., 2012). Visualization of hippocampal vasculature with biotinylated lycopersicon esculentum (tomato) lectin revealed increases in vascular area after 12 or 16 weeks HFD (Fig. $1 F, G$; for vascular area: $F_{(1,24)}$ $=8.08, p<0.01$; for tortuosity: $\left.F_{(1,24)}=5.82, p<0.05\right)$. When considered alongside of the endocrine data, these measures of vascular function and structure indicate that obesity increases 

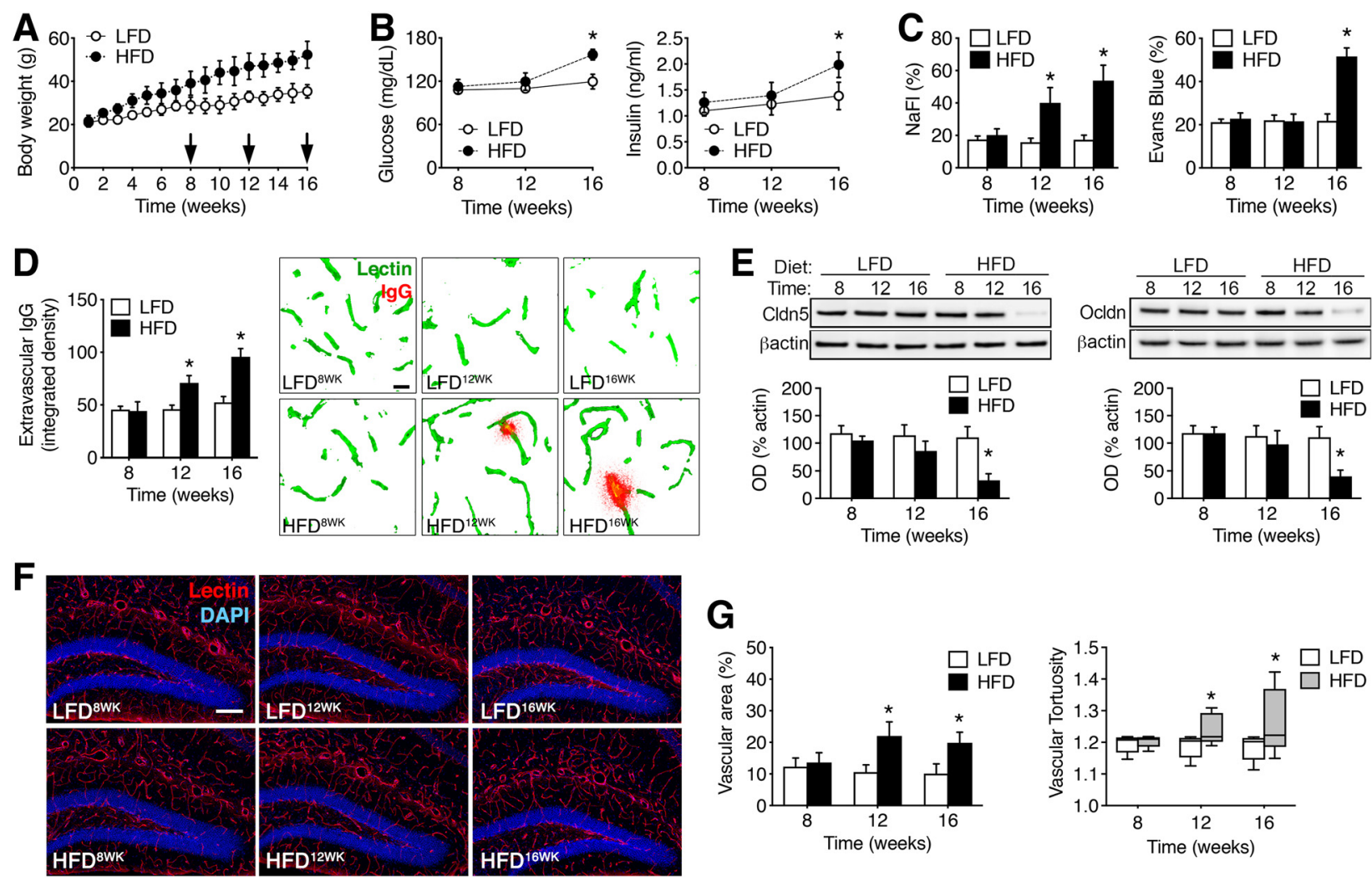

Figure 1. Progressive changes in $B B B$ integrity with increasing durations of obesogenic diet consumption. $A$, Weight gain with exposure to a HFD or LFD in male mice. $B$, Fasting hyperglycemia (left) and fasting hyperinsulinemia (right) were evident after 16 weeks consumption of HFD. C, Increased penetration of the low molecular weight tracer NaFl was observed in clarified hippocampal lysates after 12 or 16 weeks HFD (left graph). Right graph shows increases in hippocampal Evans blue after 16 weeks HFD. D. Accumulation of extravascular lgG deposits occurs in the dentate molecular layer after 12 and 16 weeks HFD. Micrographs (right) show extravascular lgG immunoreactivity after background subtraction, as described previously (Bell et al., 2010). Scale bar: (in $\mathrm{LFD}^{8 \mathrm{WK}}$ ) for all panels, $10 \mu \mathrm{m}$. $\boldsymbol{E}$, Reductions in hippocampal claudin-5 (left) and occludin (right) protein were detected by Western blotting after 16 weeks of HFD. $\boldsymbol{F}$, Micrographs show representative images of tomato lectin labeling for the indicated diets and time points. Scale bar: (in LFD ${ }^{12 W K}$ ) for all micrographs, $100 \mu \mathrm{m}$. G, Increases in vascular area (left) and tortuosity (right) in the dentate molecular layer with increasing durations of HFD. For all graphs, bar or symbol height represents the mean of $(n=4-6)$ mice per diet at each time point and error bars represent SEM. *Indicates significant differences $(p<0.05)$ determined by ANOVA.

BBB permeability and diet-induced insulin resistance promotes BBB breakdown.

\section{Disruption of the neurovascular unit in diet-induced insulin resistance}

The "neurovascular unit" includes endothelial cells and pericytes at the luminal surface, and astrocytic endfeet surrounding the abluminal surface. We used ssTEM to analyze the 3D ultrastructure of the neurovascular unit in the dentate middle molecular layer. For these experiments, a single capillary from each animal was followed through $\sim 200$ serial sections ( $n=3$ mice per diet; Fig. 2A,B). Capillaries were identified by their diameter $(2.0-6.0$ $\mu \mathrm{m})$, circularity $(\mathrm{max} / \mathrm{min}$ diameter ratio $<1.5)$, and by the absence of vascular smooth muscle cell coverage. The ssTEM dataset was generated in parallel with single-section ultrastructural measures from $(4-8)$ vessels per mouse. In mice with dietinduced insulin resistance, capillaries were frequently encircled by swollen astroglial endfeet (Fig. 2A,B). Manual segmentation of processes in direct apposition to the outer capillary basement membrane revealed significant increases in the volume of astroglial endfeet on serial micrographs (Fig. 2C; Mann-Whitney's $U=12.68, p<0.01$ ). There was no effect of diet on total capillary volume in the $3 \mathrm{D}$ dataset $\left(\mu \mathrm{m}^{3}\right.$, mean \pm SEM: $\mathrm{LFD}=2.20 \pm$ 0.18 ; HFD $=2.17 \pm 0.10$ ) or on capillary area in the $2 \mathrm{D}$ dataset $\left(\mathrm{nm}^{2}\right.$, mean \pm SEM: LFD $\left.=11.4 \pm 1.5 ; \mathrm{HFD}=10.11 \pm 1.7\right)$. However, there was substantial variability between vessels from the same animal, as reflected by within-animal SDs that exceeded $10 \%$ of per-animal averages for all samples. To control for differences in the size of individual vessels, the area of astroglial endfoot processes was expressed relative to capillary areas on a per-section basis for the $3 \mathrm{D}$ and $2 \mathrm{D}$ datasets. The ratio of endfoot process area to capillary area was significantly increased in vessels from HFD mice, consistent with perivascular astroglial swelling (Fig. $2 D ; t_{(4)}=14.88, p<0.01$ ).

Analysis of capillary ultrastructure revealed involution and atrophy of tight junctions in HFD mice (Fig. 2E). Tight junction atrophy in HFD mice was evident based on reductions in total volume in the $3 \mathrm{D}$ dataset (Fig. $2 E$; $t_{(4)}=12.28, p<0.01$ ), and based on reductions in the proportional area of tight junctions relative to endothelial cell areas in the combined dataset (Fig. 2F; $\left.t_{(4)}=15.12, p<0.01\right)$. Individual tight junctions in the $3 \mathrm{D}$ dataset traveled through (71-194) serial sections, and exhibited morphological heterogeneity across the $z$-axis (Fig. 2A,B). The density of endothelial tight junctions was significantly reduced in HFD mice, based on lower numbers of tight junctions relative to capillary length $(\mathrm{TJs} / \mu \mathrm{m}$, mean $\pm \mathrm{SEM}$; LFD $=0.42 \pm 0.05$; HFD $\left.=0.23 \pm 0.03 ; t_{(4)}=3.08, p<0.05\right)$. Capillaries from HFD mice also exhibited frequent transcytotic vesicles that were rarely observed in lean mice (Fig. $2 A, B$ ).

Pericyte coverage was significantly reduced in mice with dietinduced insulin resistance, relative to lean mice (Fig. 2G). HFD mice had smaller pericyte volumes in the $3 \mathrm{D}$ dataset (Fig. $2 G$; $t_{(4)}$ 

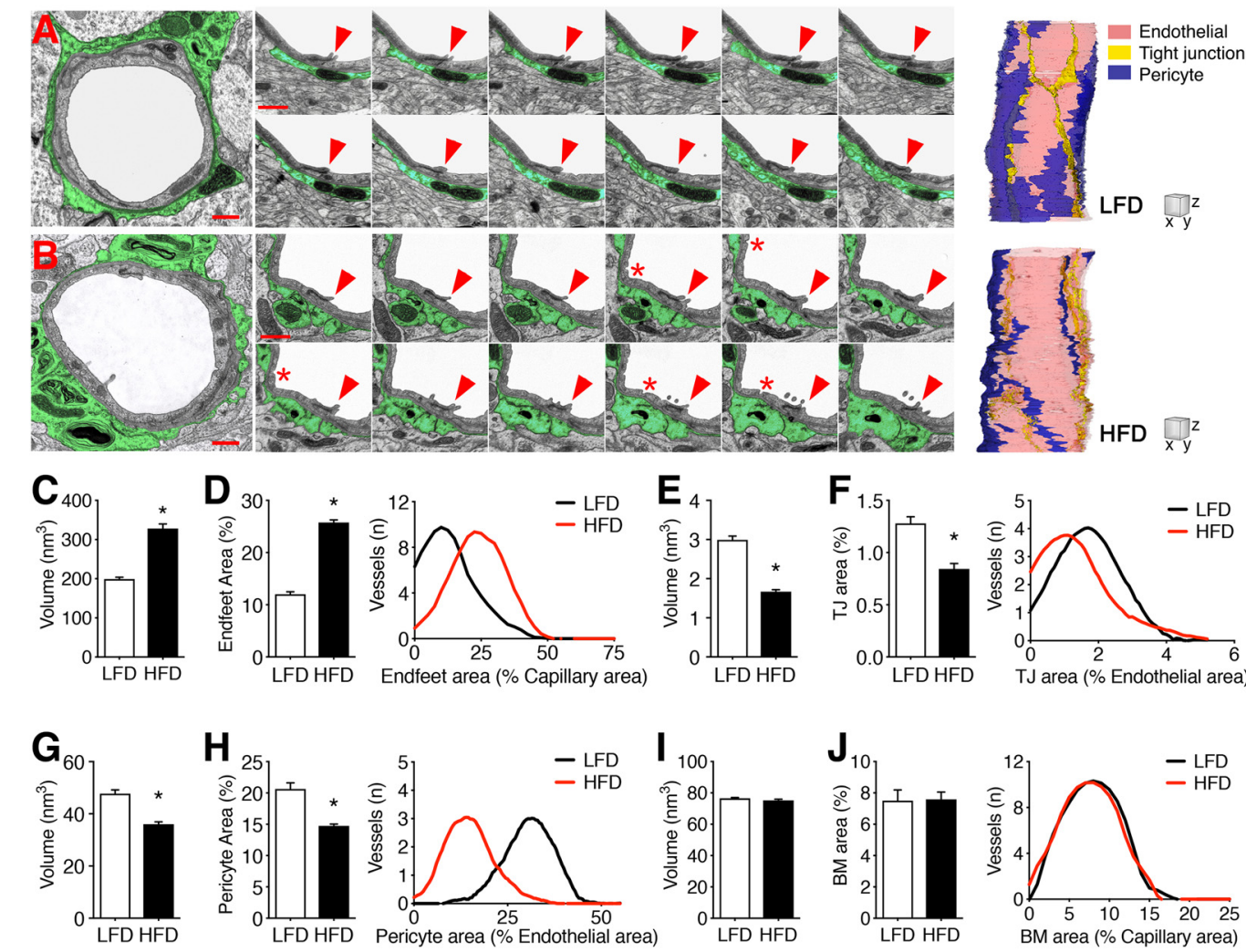

Figure 2. Three-dimensional ultrastructure of $B B B$ breakdown in mice with diet-induced insulin resistance. $A, B$, Transmission electron micrographs of representative capillaries from the hippocampal dentate molecular layer of mice on LFD $(\boldsymbol{A})$ or HFD (B) for 16 weeks. Perivascular astrocyte processes are highlighted in green. Scale bar, $1.0 \mu \mathrm{m}$. Cropped images (middle) show serial sections through a single tight junction (red arrow) between two adjacent endothelial cells in mice on LFD (A) or HFD. For serial sections: scale bar, $0.5 \mu \mathrm{m}(z=0.6 \mu \mathrm{m}) . \boldsymbol{B}$, Transcytotic vesicles (red asterisks) were frequently observed in capillaries from HFD mice. Far right, $3 \mathrm{D}$ reconstructions of representative capillaries from mice on LFD or HFD. For reconstructions: scale cube (XYZ), $1.0 \mu \mathrm{m}$. C, Increases in astroglial endfoot process volume in serial images from HFD mice. D, Bar graph shows increases in astroglial endfoot area relative to total capillary area in serial and single-section analysis. Histogram shows data for individual vessels. $\boldsymbol{E}$, Endothelial tight junctions (TJs) exhibit significant atrophy in HFD mice based on smaller volumes in serial images. $\boldsymbol{F}$, Bar graph shows reductions in the area of tight junctions relative to endothelial cell areas in serial and single-section analysis. Histogram shows data for individual vessels. $\mathbf{G}$, Pericyte coverage is significantly reduced in HFD mice, based on smaller pericyte volumes in $3 \mathrm{D}$ analysis. $\boldsymbol{H}$, Bar graph shows reductions in the ratio of pericyte area to endothelial cell area in the combined $2 \mathrm{D}$ and $3 \mathrm{D}$ dataset. Histogram shows data for individual vessels. $I$, The volume of the endothelial basement membrane (BM) is comparable between LFD and HFD mice.J, The proportional volume of the basement membrane relative to capillary area is unaffected by diet. For graphs in $\boldsymbol{C}, \boldsymbol{E}, \boldsymbol{G}$, and $\boldsymbol{I}$, bar height represents the average of three capillaries traced through $\sim 200$ serial sections from $(n=3)$ mice per diet. For graphs in $D, F, H$, and $J$, bar height represents the average of $(5-9)$ vessels from single- and serial-section analysis of $(n=3)$ mice per diet. For all graphs, error bars represent the SEM and asterisks $(*)$ denote statistical significance at $p<0.05$ following $t$ test or Mann-Whitney's $U$ test.

$=6.80, p<0.05)$, and exhibited reductions in pericyte area as a proportion of capillary area in the combined dataset (Fig. $2 \mathrm{H} ; t_{(4)}$ $=17.27, p<0.01)$. Although ultrastructural alterations were observed in both endothelial cells and pericytes, the anatomy of the capillary basement membrane was intact (Fig. $2 I, J ; t_{(4)}=$ 0.57 , n.s.), and there was no evidence of atrophy at the pericyte basement membrane (volume, $\mathrm{nm}^{3}$, mean \pm SEM; LFD $=$ $14.73 \pm 0.44 ; \mathrm{HFD}=15.21 \pm 0.48$ ). Additionally, the average lumen diameter was unaffected by diet (lumen diameter, $\mu \mathrm{m}$, mean \pm SEM; LFD $=3.94 \pm 0.20$; HFD $=3.83 \pm 0.21)$. Together, these data indicate that diet-induced insulin resistance promotes pericyte regression and compromises tight junctions among capillaries in the hippocampal dentate gyrus.

\section{Adora2a antagonism rescues BBB integrity and LTP in diet-induced insulin resistance}

Systemic administration of Adora2a agonists increase BBB permeability, and Adora2a antagonism protects the BBB in experimental autoimmune disease models (Mills et al., 2008; Carman et al., 2011). We administered the selective Adora2a antagonist SCH58261 (0.1 mg/kg, i.p.; every $12 \mathrm{~h}$ for $3 \mathrm{~d})$. Twelve hours after the final injection of SCH58261, mice were injected with NaFl and Evans blue for analysis of BBB permeability. Treatment with SCH58261 eliminated the effects of HFD on hippocampal NaFl and Evans blue concentrations (Fig. $3 A-C$; for NaFl: $F_{(1,32)}=$ 14.37, $p<0.01$; for Evans blue: $F_{(1,32)}=12.60, p<0.01$ ). There was no effect of diet or SCH58261 on serum tracer concentrations $(\mathrm{NaFl}, \mathrm{mg} / \mathrm{ml}$, mean $\pm \mathrm{SEM}: \mathrm{LFD} /$ Vehicle $=8.7 \pm 0.5 ; \mathrm{LFD} /$ SCH58261 $=9.5 \pm 0.3 ; \mathrm{HFD} /$ Vehicle $=9.1 \pm 0.6 ; \mathrm{HFD} /$ SCH58261 $=9.9 \pm 0.5$; Evans blue, $\mathrm{mg} / \mathrm{ml}$, LFD/Vehicle $=$ $40.4 \pm 1.2 ; \mathrm{LFD} / \mathrm{SCH} 58261=40.7 \pm 1.1 ; \mathrm{HFD} /$ Vehicle $=41.2 \pm$ 1.1; HFD/SCH58261 $=40.9 \pm 0.8$ ). Extravasation of NaFl and Evans blue was also evident on hippocampal sections from vehicle-treated HFD mice, but not SCH58261-treated mice (Fig. 3B).

Food intake and body weight were monitored to determine whether SCH58261 would induce weight loss. There was no effect of SCH58261 on body weight (weight in g, mean \pm SEM: LFD/ Vehicle $=38.1 \pm 0.8 ; \mathrm{LFD} / \mathrm{SCH} 58261=36.4 \pm 1.0 ; \mathrm{HFD} /$ Vehi$\mathrm{cle}=46.7 \pm 1.4 ; \mathrm{HFD} / \mathrm{SCH} 58261=48.1 \pm 0.9$ ), and there was also no effect on food intake during the $72 \mathrm{~h}$ treatment period $(\mathrm{kCal} / 24 \mathrm{~h}$, mean $\pm \mathrm{SEM}: \mathrm{LFD} /$ Vehicle $=11.5 \pm 0.2 ; \mathrm{LFD} /$ SCH58261 $=10.9 \pm 0.4 ; \mathrm{HFD} /$ Vehicle $=12.0 \pm 0.3 ; \mathrm{HFD} /$ SCH58261 $=11.8 \pm 0.2$ ). Postprandial serum glucose levels were 
A
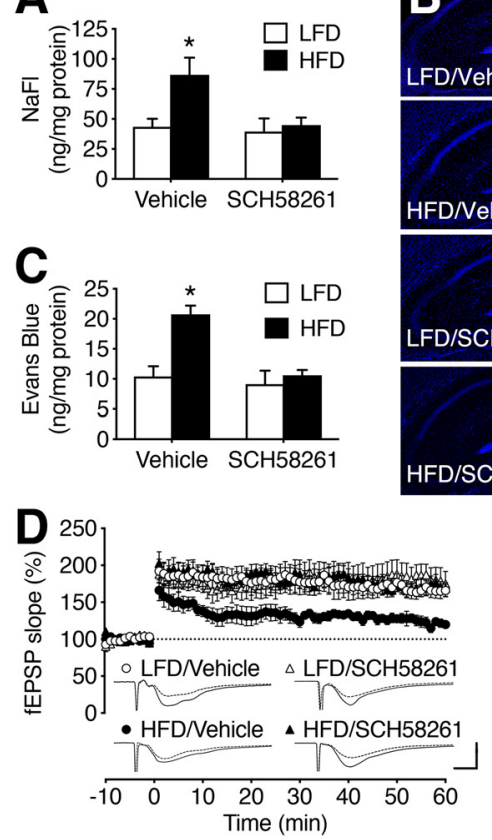
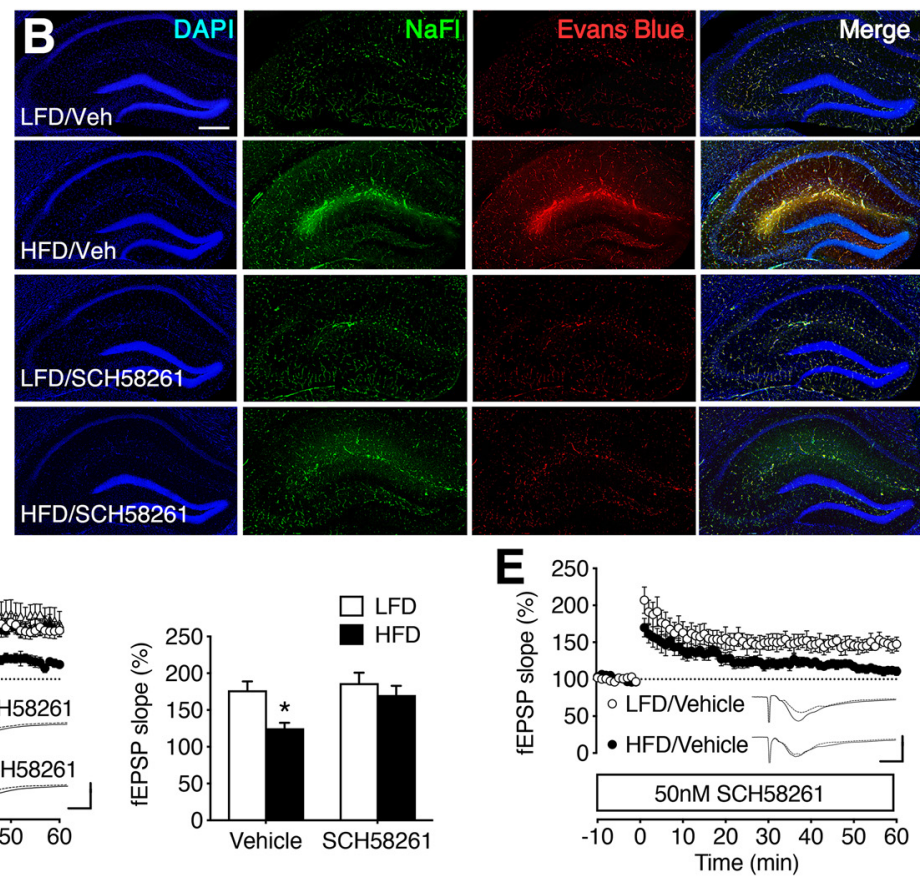

Figure 3. Adora2a antagonism restores BBB integrity and rescues synaptic plasticity in mice with diet-induced insulin resistance. $A$, Increases in hippocampal NaFl extravasation were eliminated by treatment with the Adora2a antagonist SCH58261 or vehicle (Veh) in mice maintained on LFD or HFD for 16 weeks. $\boldsymbol{B}$, Visualization of NaFl and Evans blue on hippocampal sections. Scale bar: (in LFD/Veh) for all micrographs, $200 \mu \mathrm{m}$. C, Increased hippocampal penetration of Evans blue was blocked by SCH58261 in mice with diet-induced insulin resistance. $\boldsymbol{A}$, $\boldsymbol{C}$, Bar height represents the average of $(n=8-12)$ mice per condition. $\boldsymbol{D}$, Adora2a antagonist treatment normalizes dentate gyrus long-term potentiation in hippocampal slices from HFD mice. Bar graph (right) shows percentage increase in field EPSP (fEPSP) slope during minutes 50-60 of recording. $\boldsymbol{E}$, Bath application of SCH58261 has no effect on LTP deficits in slices from vehicle-treated HFD mice. $\boldsymbol{D}$, $\boldsymbol{E}$, Bars or symbol height represents the average of $(n=9-14)$ slices from each condition. For traces in $\boldsymbol{D}$ and $\boldsymbol{E}$, scale bars: (bottom right) $y$-axis, $1.0 \mathrm{mV}$; $x$-axis, $10 \mathrm{~ms}$. For all graphs, error bars represent SEM and asterisk $\left(^{*}\right)$ indicates significance at $p<0.05$ by ANOVA with Bonferroni's post hoc.

also unaffected by SCH58261 (mg/dl, mean \pm SEM: LFD/Vehicle $=128.3 \pm 6.2 ; \mathrm{LFD} / \mathrm{SCH} 58261=134.8 \pm 7.3$; HFD/Vehicle $=185.3 \pm 8.8 ;$ HFD/SCH58261 = 178.2 \pm 6.9$)$, indicating that systemic inhibition of Adora2a restored BBB integrity without influencing metabolic dysfunction.

Diet-induced insulin resistance impairs hippocampal longterm potentiation in rodent models (Stranahan et al., 2008; Hao et al., 2016). To examine whether reinstatement of BBB integrity with systemic Adora2a antagonism would reverse LTP deficits, we performed electrophysiological recordings of dentate gyrus LTP in hippocampal slices. Systemic treatment with SCH58261 rescued LTP in HFD mice, without influencing LTP in slices from LFD mice (Fig. 3D; $F_{(1,26)}=7.43, p<0.05$ ). Because peripheral administration of SCH58261 also suppresses neuronal Adora2a activation (Fredholm et al., 1998), restoration of LTP could be independent of changes in vascular permeability. To address this possibility, slices from vehicle-treated mice were incubated with $50 \mathrm{~nm}$ SCH58261, with continuous application throughout recording. Direct application of SCH58261 had no effect on LTP deficits in HFD mice (Fig. $3 E$; $t_{(11)}=5.10, p<0.01$ ), suggesting that the non-neuronal effects of Adora2a antagonism might contribute to functional rescue following systemic administration.

\section{Generation and characterization of transgenic mice with inducible resistance to $\mathrm{BBB}$ opening}

We generated transgenic mice with inducible ablation of endothelial Adora2a by crossing Tie ${ }^{\text {rtTA }}$ mice with mice bearing loxP sites flanking exon 2 of Adora2a (Adora $2 \mathrm{a}^{\mathrm{fl} / \mathrm{fl}}$ ). The resultant Tie $2^{\text {rtTA }} /$ Adora $2 \mathrm{a}^{\mathrm{fl} / \mathrm{fl}}$ mice were crossed with mice that express cre recombinase under a tetracycline-On promoter element $\left(\right.$ tetO $\left.^{\text {cre }}\right)$ to generate mice with inducible ablation of Adora2a in endothelial cells (Fig. 4A). To examine selectivity, genomic DNA from whole hippocampus, BVECs, primary microglia, and primary astrocytes was amplified with primers targeted to the Adora2a deleted sequence. Minimal amplification was observed in BVECs from AR2ATg mice, and robust amplification was observed in all other cell types and tissues, consistent with selective deletion in endothelial cells (Fig. 4B).

\section{Endothelial Adora2a increases BBB permeability and} promotes pericyte regression in diabetic mice

To investigate resistance to BBB leakiness in diet-induced obesity, transgenic $(\mathrm{Tg})$ mice and $\mathrm{nTg}$ littermates were maintained on HFD or LFD for 16 weeks, with induction during Week 12 of the experiment. Food intake and body weight were monitored to determine whether endothelial ablation of Adora2a would induce weight loss. Both Tg and nTg HFD mice had higher body weights than LFD mice, and there was no effect of endothelial AR2AKO on body weight (Fig. $4 C$; effect of diet: $F_{(1,28)}=9.70$, $p<0.01$; effect of genotype: n.s.). There was also no effect of genotype on food intake $(\mathrm{kCal} / \mathrm{d}$, mean $\pm \mathrm{SEM}: \mathrm{nTg} / \mathrm{LFD}=$ $13.4 \pm 1.9 ; \mathrm{Tg} / \mathrm{LFD}=12.2 \pm 1.6 ; \mathrm{nTg} / \mathrm{HFD}=12.9 \pm 1.1 ; \mathrm{Tg} /$ $\mathrm{HFD}=13.6 \pm 1.3)$. Tg mice and $\mathrm{nTg}$ littermates developed comparable deficits in glycemic control on HFD (Fig. 4D; effect of diet: $F_{(1,22)}=12.71, p<0.01$; effect of genotype: $n$.s.), indicating that selective ablation of endothelial Adora2a did not confer resistance to metabolic dysfunction.

Analysis of hippocampal tight junction protein expression revealed that the effects of diet-induced insulin resistance were dependent on endothelial Adora2a expression (Fig. 4E). Tg mice were protected against reductions in claudin-5 $\left(F_{(1,20)}=5.77\right.$, $p<0.05)$ and occludin $\left(F_{(1,20)}=6.34, p<0.01\right)$ on HFD. There 
A
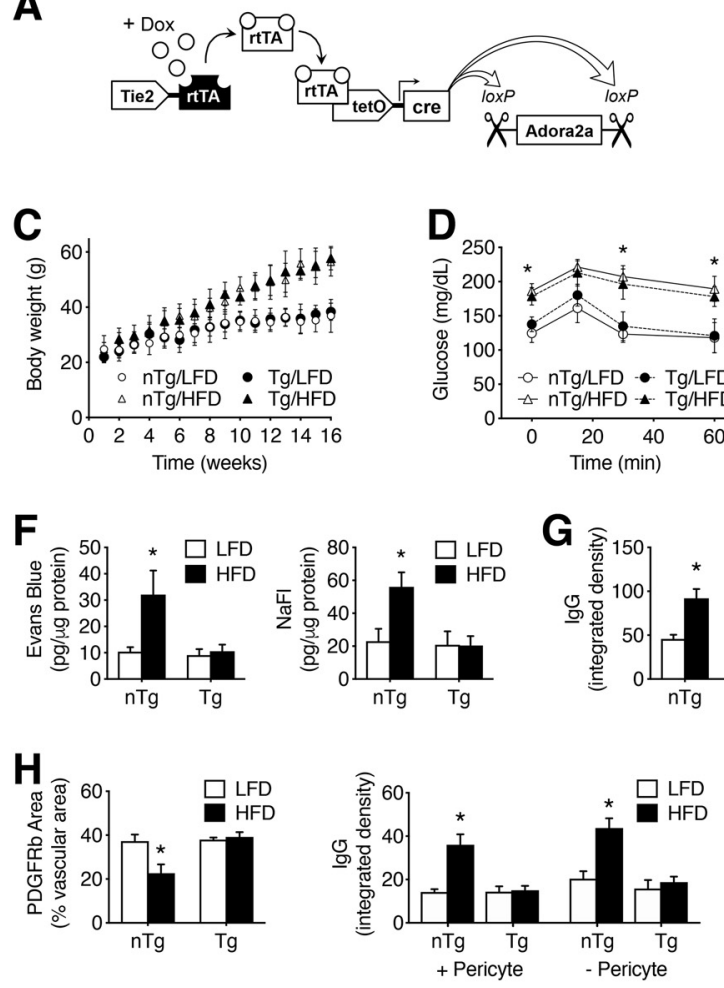

B

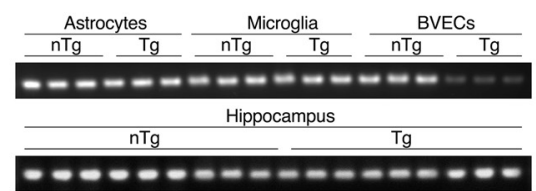

E
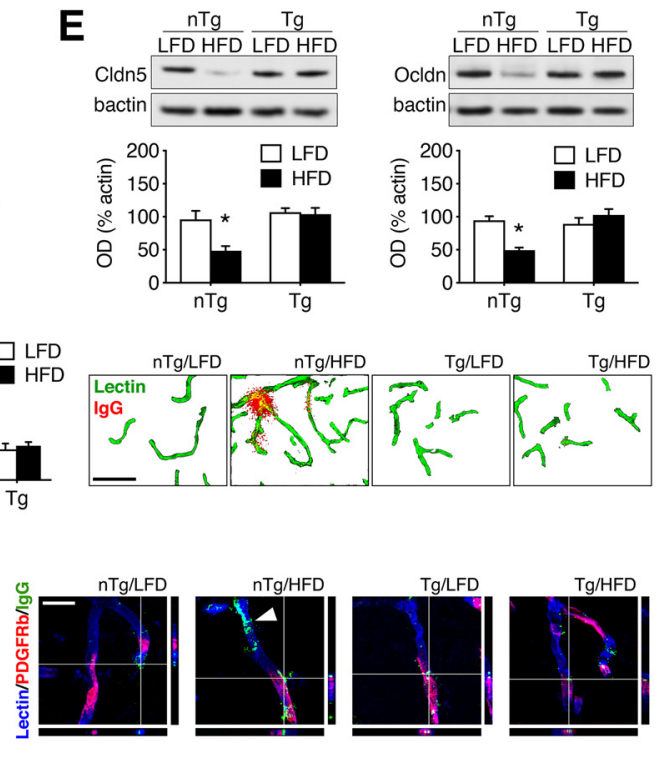

Figure 4. Inducible ablation of Adora2a on endothelial cells maintains BBB structure and function in mice with diet-induced insulin resistance. $A$, Schematic depiction of Tg mouse model with inducible ablation of Adora2a in Tek/Tie2-expressing endothelial cells. B, Amplification of genomic DNA from BVECs, microglia, astrocytes, or whole hippocampus with primers directed against the

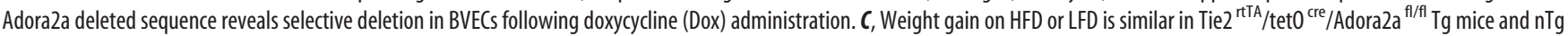
littermates, with no differences either before and after induction during Week 12.D, Intraperitoneal glucose tolerance testing revealed comparable deficits in glycemic control in Tg/HFD mice and nTg/HFD littermates. $\boldsymbol{C}, \boldsymbol{D}$, Symbol height represents the average of $(n=14-18)$ mice per condition. $\boldsymbol{E}$, Inducible ablation of endothelial Adora2a restores hippocampal expression of the tight junction proteins claudin-5 (left) and occludin (right). Bar height represents the average of $(n=4-6)$ mice per condition. $\boldsymbol{F}$, Increases in NaFl (left) and Evans blue (right) extravasation were eliminated in Tg mice on HFD. Bar height shows the mean of $(n=8-12)$ mice per condition. $G$, Tg mice are protected against extravascular lgG accumulation with HFD. Micrographs (right) show immunofluorescence detection of extravascular lgG and tomato lectin after background subtraction. Scale bar, $25 \mu \mathrm{m}$. $\boldsymbol{H}$, Ablation of endothelial Adora2a eliminates obesity-induced pericyte regression (left). Right graph shows perivascular lgG deposits in microvascular regions with or without PDGFRb + pericytes. Micrographs (far right) show labeling for PDGFRb, tomato lectin, and mouse lgG. Scale bar, $5 \mu \mathrm{m} . \mathbf{G}, \boldsymbol{H}$, Bar height represents the average of $(n=4-6)$ mice per condition. For all graphs, error bars represent SEM and asterisk $\left(^{*}\right)$ indicates significance at $p<0.05$ by ANOVA with Bonferroni's post hoc.

were no differences in tight junction protein expression between $\mathrm{Tg}$ and $\mathrm{nTg}$ mice on LFD (Fig. 4E). Transgenic mice were also protected against hippocampal extravasation of Evans blue and NaFl on HFD (Fig. 4F; for NaFl: $F_{(1,20)}=8.64, p<0.01$; for Evans blue: $\left.F_{(1,20)}=7.90, p<0.01\right)$, indicating that increased vascular permeability with diet-induced insulin resistance requires endothelial Adora2a expression. There was no effect of diet or genotype on serum concentrations of $\mathrm{NaFl}(\mathrm{mg} / \mathrm{ml}$, mean \pm SEM: $\mathrm{nTg} / \mathrm{LFD}=8.5 \pm 0.6 ; \mathrm{nTg} / \mathrm{HFD}=9.2 \pm 0.6 ; \mathrm{Tg} / \mathrm{LFD}=9.1 \pm$ $0.8 ; \mathrm{Tg} / \mathrm{HFD}=9.0 \pm 0.4)$ or Evans blue $(\mathrm{mg} / \mathrm{ml}$, mean \pm SEM: $\mathrm{nTg} / \mathrm{LFD}=42.9 \pm 1.7 ; \mathrm{nTg} / \mathrm{HFD}=44.1 \pm 2.3 ; \mathrm{Tg} / \mathrm{LFD}=$ $40.8 \pm 1.6 ; \mathrm{Tg} / \mathrm{HFD}=43.0 \pm 1.5$ ). We visualized extravascular IgG accumulation by immunofluorescence as an additional measure of BBB leakiness (Fig. 4G). nTg HFD mice exhibited significant increases in IgG immunoreactivity in the dentate molecular layer, but $\mathrm{Tg} / \mathrm{HFD}$ mice exhibited very limited extravascular IgG labeling (Fig. $4 G ; F_{(1,20)}=8.99, p<0.01$ ). There were no differences between $\mathrm{Tg}$ and $\mathrm{nTg}$ mice on LFD, consistent with a role for endothelial Adora2a in diet-induced BBB leakiness.

In the ultrastructural experiments, we observed that pericyte regression coincided with the onset of $\mathrm{BBB}$ breakdown in $\mathrm{Wt}$ mice on HFD (Fig. 2). To examine whether endothelial Adora2a ablation influences pericyte coverage, we performed immunofluorescence labeling for the pericyte marker PDGFRb and tomato lectin (Fig. 4H). Transgenic mice were completely protected against reductions in pericyte coverage with HFD feeding, based on similar ratios between PDGFRb labeling area and total vascular area in comparison with $\mathrm{nTg} / \mathrm{LFD}$ mice (Fig. $4 H ; F_{(1,20)}=$ $13.55, p<0.01)$. Because the CNS microvasculature exhibits domain-specific IgG leakage at sites distal to the closest pericyte (Bell et al., 2010), we examined perivascular IgG accumulation as a function of proximity to the nearest pericyte (Fig. $4 H$ ). This analysis revealed that $\mathrm{nTg} / \mathrm{HFD}$ mice exhibit comparable increases in perivascular IgG in microvascular regions where pericytes are present, and in regions lacking pericyte coverage (Fig. $\left.4 H ; F_{(1,20)}=5.02, p<0.01\right)$. By contrast, Tg/HFD mice exhibited very limited perivascular IgG immunoreactivity, and this did not vary as a function of pericyte coverage (Fig. 4H; post hoc comparison with $\mathrm{nTg} / \mathrm{LFD}$, n.s.).

\section{Adora2a-mediated BBB breakdown disrupts perivascular astroglia in diet-induced insulin resistance}

Our ultrastructural studies revealed that HFD consumption promotes swelling of astroglial endfeet in Wt mice (Fig. 2). To examine astroglial responses in $\mathrm{Tg}$ mice with protection against obesity-induced BBB breakdown, we performed immunofluorescence labeling for the astroglial marker GFAP on hippocampal sections (Fig. 5A). Analysis of GFAP immunoreactivity in the 

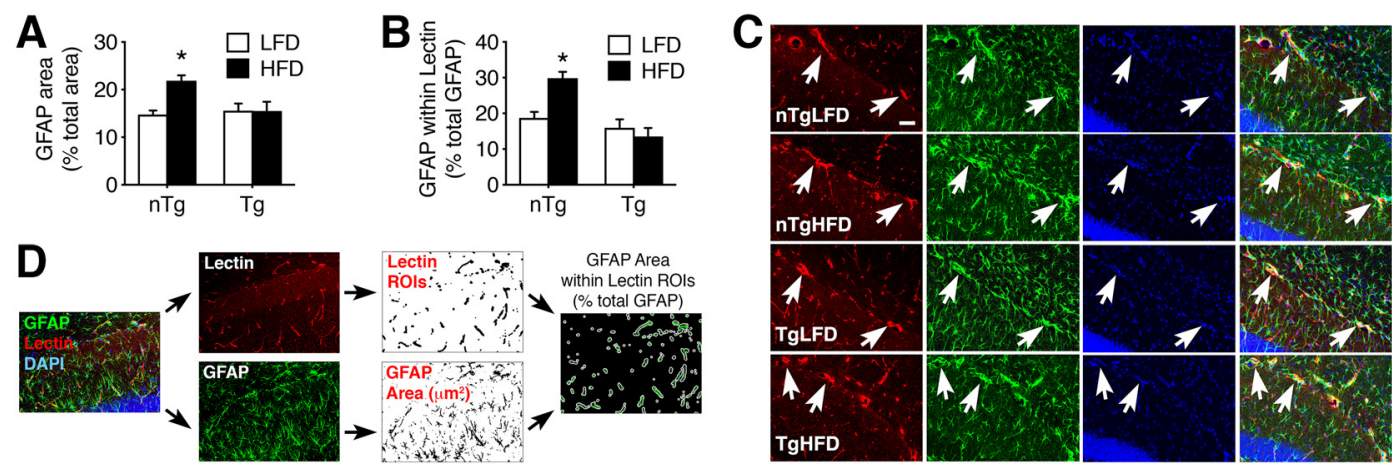

Figure 5. Inducible ablation of endothelial Adora2a prevents high-fat diet-induced astrogliosis and maintains perivascular astroglial processes. $A$, Ablation of endothelial Adora2a eliminates astrogliosis in mice with diet-induced insulin resistance. $\boldsymbol{B}$, Increases in perivascular GFAP labeling in HFD mice are dependent on endothelial Adora2a activation. $\boldsymbol{C}$, Representative micrographs of tomato lectin and GFAP labeling in the dentate molecular layer. Arrows indicate perivascular astroglial processes. Scale bar shown for nTg/LFD $=20$ microns and applies to all panels. $D$, Schematic depiction of method for analysis of perivascular GFAP. For all graphs, bar height represents the mean of $(n=6-8)$ mice per condition, error bars represent SEM and asterisk (*) indicates significance at $p<0.05$ by ANOVA with Bonferroni's post hoc.

dentate molecular layer revealed widespread astrogliosis in $\mathrm{nTg} /$ HFD mice with diet-induced insulin resistance (Fig. $5 A, B ; F_{(1,25)}$ $=7.04, p<0.05)$. Increases in GFAP immunoreactivity were not evident in sections from Tg/HFD mice, which were indistinguishable from $\mathrm{nTg} / \mathrm{LFD}$ mice (Fig. $5 A, B$ ). Parallel visualization of hippocampal vasculature with tomato lectin was used to delineate regions of interest for analysis of perivascular astroglial processes (Fig. 5C,D). Perivascular astroglial processes, as defined by GFAP labeling, were more frequent in nTg/HFD mice than in all other groups (Fig. $5 C ; F_{(1,25)}=9.74, p<0.01$ ), potentially implicating endothelial Adora2a activation in localized reactive astrogliosis due to diet-induced insulin resistance. Because there were no genotype differences between mice on LFD, it is likely that Adora2a-mediated BBB breakdown disrupts the structure and function of the hippocampal astroglia in diet-induced insulin resistance.

\section{Adora2a promotes cerebrovascular inflammation in mice with diet-induced insulin resistance}

The functional role of cerebrovascular endothelial cells is not limited to barrier maintenance and permeability. To gain insight into potential differences in vascular inflammation on HFD, we performed flow cytometric experiments in isolated cerebral MVs. The endothelial activation markers E-selectin/CD62E and ICAM-1 were quantified in SSClow/FSCmid/CD45-/CD31+ cells, as shown (Fig. 6A). Induction of ICAM-1 was significantly increased among CD45-/CD31+ cells from the brains of nTg/ HFD mice, but there was no increase in cells isolated from $\mathrm{Tg} /$ HFD mice (Fig. $6 B ; F_{(1,28)}=7.61, p<0.01$ ). This pattern was also observed for e-selectin, indicating that obesity promotes vascular inflammation via an Adora2a-dependent mechanism (Fig. 6C,D; $\left.F_{(1,28)}=8.71, p<0.01\right)$.

We next performed $\mathrm{qPCR}$ analysis of gene expression in MVs to validate the suppression of target genes in Tg mice and examine a broader panel of transcripts linked with endothelial activation. Analysis of Adora1 and Adora2a revealed that HFD upregulates microvascular Adora2a in nTg mice (Fig. 6E; diet $X$ genotype interaction: $\left.F_{(1,18)}=8.08, p<0.05\right)$. Tg mice on either diet exhibited comparable reductions in Adora2a expression (effect of genotype: $F_{(1,18)}=6.94, p<0.01$, without any evidence of compensatory changes in Adora1 (Fig. 6E). After validating the changes in Adora2a, we measured claudin- 1 and junctional celladhesion motif 2 (Jam2), which encode tight junction proteins. $\mathrm{nTg}$ mice on HFD exhibited reductions in claudin-1 (Fig. 6E;
$\left.F_{(1,18)}=5.43, p<0.05\right)$ and Jam2 gene expression $\left(F_{(1,18)}=6.03\right.$, $p<0.05)$. Tg mice were protected against HFD-induced downregulation of claudin-1 and Jam2 (Fig. 6E), indicating that endothelial Adora2a activation reduces tight junction gene transcription.

Vascular endothelial growth factor (VEGF) signaling potently regulates angiogenesis, $\mathrm{BBB}$ permeability, and the function of endothelial cells and pericytes. After observing changes in tight junction gene expression, we investigated potential changes in VEGF signaling by qPCR analysis of VEGF-A, VEGF-B, and the genes encoding VEGFR1 (Flt1) and VEGFR2 (Kdr). In nTg mice, HFD was associated with increased expression of VEGF-A (Fig. $\left.6 E ; F_{(1,18)}=5.63, p<0.05\right)$ and VEGFR2 in microvessel preparations (Fig. $\left.6 E ; F_{(1,18)}=4.54, p<0.05\right)$. These effects were not observed in Tg mice on HFD, indicating that induction of VEGF-A and VEGFR2 was Adora2a-dependent (Fig. 6E). There was no effect of diet or genotype on serum levels of VEGF-A $(\mathrm{pg} / \mathrm{ml}$, mean $\pm \mathrm{SEM}: \mathrm{nTg} / \mathrm{LFD}=110.6 \pm 17.5 ; \mathrm{nTg} / \mathrm{HFD}=$ $120.9 \pm 16.5 ; \mathrm{Tg} / \mathrm{LFD}=117.3 \pm 20.2 ; \mathrm{Tg} / \mathrm{HFD}=115.4 \pm 14.8$ ). Reductions in microvascular Glutl were reported after acute HFD consumption by other groups (Jais et al., 2016). Quantification of Glut 1 gene expression revealed significant reductions in HFD mice from both genotypes (Fig. $6 E$; effect of diet: $F_{(1,18)}=$ $10.46, p<0.01)$. Changes in gene expression and cell surface marker induction in MVs were not exclusive to isolated cells, as immunofluorescence detection of COX-2 in CD31+ endothelial cells revealed similar patterns (Fig. $6 F$ ). Together, these changes in implicate Adora2a in erosion of the cerebrovascular barrier and vascular inflammation.

\section{Adora2a-mediated cerebrovascular dysfunction impairs synaptic plasticity and cognition}

To determine whether Adora2a-mediated cerebrovascular dysfunction impairs memory, Tg mice and $\mathrm{nTg}$ littermates were maintained on HFD or LFD for 16 weeks, with transgene induction during Week 12 as described for analysis of cerebrovascular inflammmation. Testing in the water maze revealed comparable acquisition of spatial memory, as determined by similar path lengths in all groups of mice (Fig. 7A). However, performance in the probe trial was significantly impaired in $\mathrm{nTg} / \mathrm{HFD}$ mice, indicative of deficits in spatial memory retention (Fig. $7 A ; F_{(1,49)}=$ $13.53, p<0.01)$. There were no differences between Tg/LFD and $\mathrm{nTg} / \mathrm{LFD}$ mice in the probe trial, and all groups of mice were able to navigate toward the visible platform (distance, meters; mean \pm 

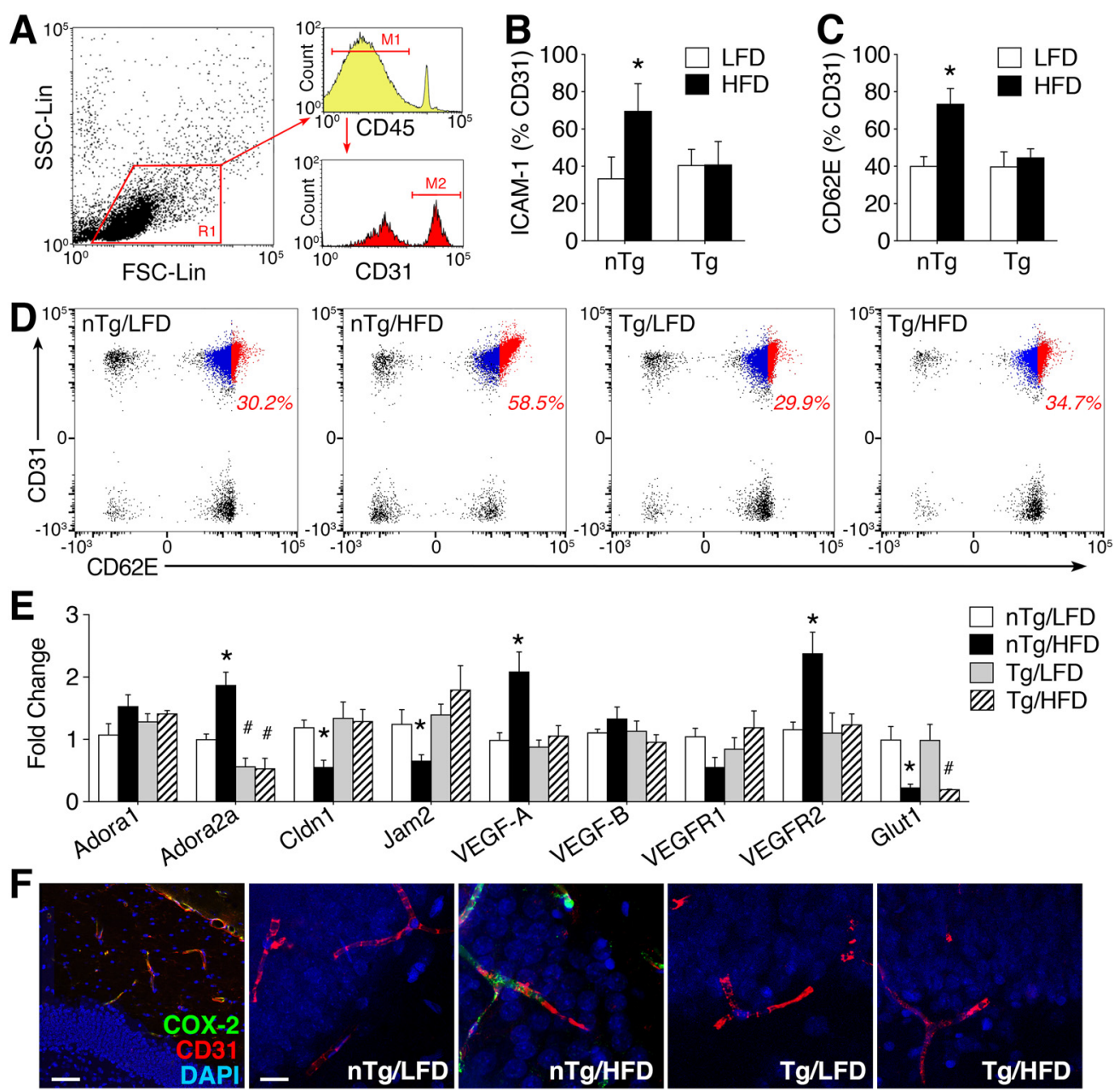

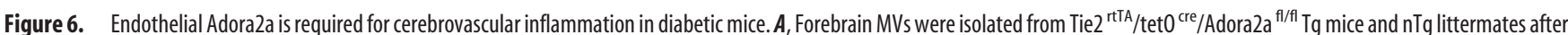
16 weeks of LFD or HFD. Cells were gated on forward and side scatter [shown in region 1 (R1)] and the activation markers e-selectin (CD62E) and ICAM-1 were measured in CD45-/CD31+ endothelial cells. For histograms, marker 1 (M1) denotes gating of CD45-negative cells and marker 2 (M2) indicates gating of CD31 + cells. B, Endothelial Adora2a ablation prevents induction of ICAM-1 in mice on HFD. C, Tg mice were protected against endothelial induction of CD62E on HFD. D, Representative scatter plots of CD62E in CD45-/CD31+ forebrain endothelial cells. Activated endothelial cells (CD31+/CD62E + ) are shown in red and quiescent endothelial cells (CD31+/CD62E - ) are shown in blue. Percentages (red text) reflect proportion of activated endothelial cells relative to total endothelial cells. E, qPCR analysis of adenosine receptor subtypes (Adora1, Adora2a), genes encoding tight junction proteins (Cldn1, Jam2), VEGF signaling genes (VEGF-A, VEGF-B, VEGFR1, VEGFR2), and glucose transporter 1 (Glut1) in MV preparations from nTg and Tg mice on HFD or LFD. $\boldsymbol{F}$, Immunofluorescence visualization of the endothelial activation marker COX-2 in hippocampal sections. Scale bars: far left, $50 \mu \mathrm{m}$; (in nTg/LFD) for all other micrographs, $10 \mu \mathrm{m} . \boldsymbol{B}, \boldsymbol{C}$, Bar height represents the average of $(n=6-8)$ mice per condition; $\boldsymbol{E}$, bar height shows the average of $(n=4-6)$ mice per condition. For all graphs, error bars represent SEM and asterisk $\left(^{*}\right)$ indicates significance at $p<0.05$ by ANOVA with Bonferroni's post hoc.

SEM: $\mathrm{nTg} / \mathrm{LFD}=2.5 \pm 0.38 ; \mathrm{Tg} / \mathrm{LFD}=2.4 \pm 0.39 ; \mathrm{nTg} / \mathrm{HFD}=$ $2.3 \pm 0.41 ; \mathrm{Tg} / \mathrm{HFD}=2.4 \pm 0.26$ ).

The water maze paradigm relies on aversive motivation and typically results in minor weight loss, which did not differ between groups in the current experiment (data not shown). However, to offset any potential confounds associated with physiological stress, mice were also tested in the novel object preference and Y-maze paradigms. In the novel object preference test, $\mathrm{nTg} / \mathrm{HFD}$ mice performed close to chance, spending $\sim 50 \%$ of time with the novel object at all post-training time points (Fig. $7 B$; diet $\times$ genotype interaction: $\left.F_{(3,82)}=15.14, p<0.01\right)$. Transgenic mice preferentially explored the novel object and performed on par with nTg/LFD mice (Fig. 6B). All groups of mice spent similar amounts of time interacting with the two stimulus objects (data not shown). In the Y-maze, nTg/HFD mice exhibited significant reductions in spontaneous alternation (Fig. $7 C$; diet $\times$ genotype interaction: $\left.F_{(1,29)}=6.25, p<0.05\right)$. Inducible ablation of endothelial Adora2a restored spontaneous alternation in Tg/HFD mice (Fig. 7C). There were no differences in alternation or object recognition between $\mathrm{nTg} / \mathrm{LFD}$ and $\mathrm{Tg} / \mathrm{LFD}$ mice, consistent with the pattern observed in the water maze.

We next measured LTP at medial perforant path synapses on dentate gyrus granule neurons, which are located in the same highly vascularized portion of the middle molecular examined in the ultrastructural experiments. Adora2a Tg mice were protected against HFD-induced LTP deficits, based on normalization of fEPSP slopes during minutes $50-60$ of recording (Fig. $7 D, E$; diet $\times$ genotype interaction: $\left.F_{(1,25)}=6.38, p<0.05\right)$. There were no differences in LTP between Tg and nTg mice on LFD (Fig. 7E), indicating that endothelial Adora2a ablation had no effect on plasticity in non-obese mice. Presynaptic paired-pulse depression was unaffected by HFD or genotype (Fig. $7 F$ ), and input/ output ratios were also comparable across all groups (Fig. $7 G$ ). Dendritic spines are the primary postsynaptic site for excitatory neurotransmission, and to examine the structural basis for LTP deficits, we quantified spines in the same anatomical region used for electrophysiological recordings. Endothelial Adora2a ablation maintained dendritic spine density in HFD mice (Fig. $7 \mathrm{H}$, 

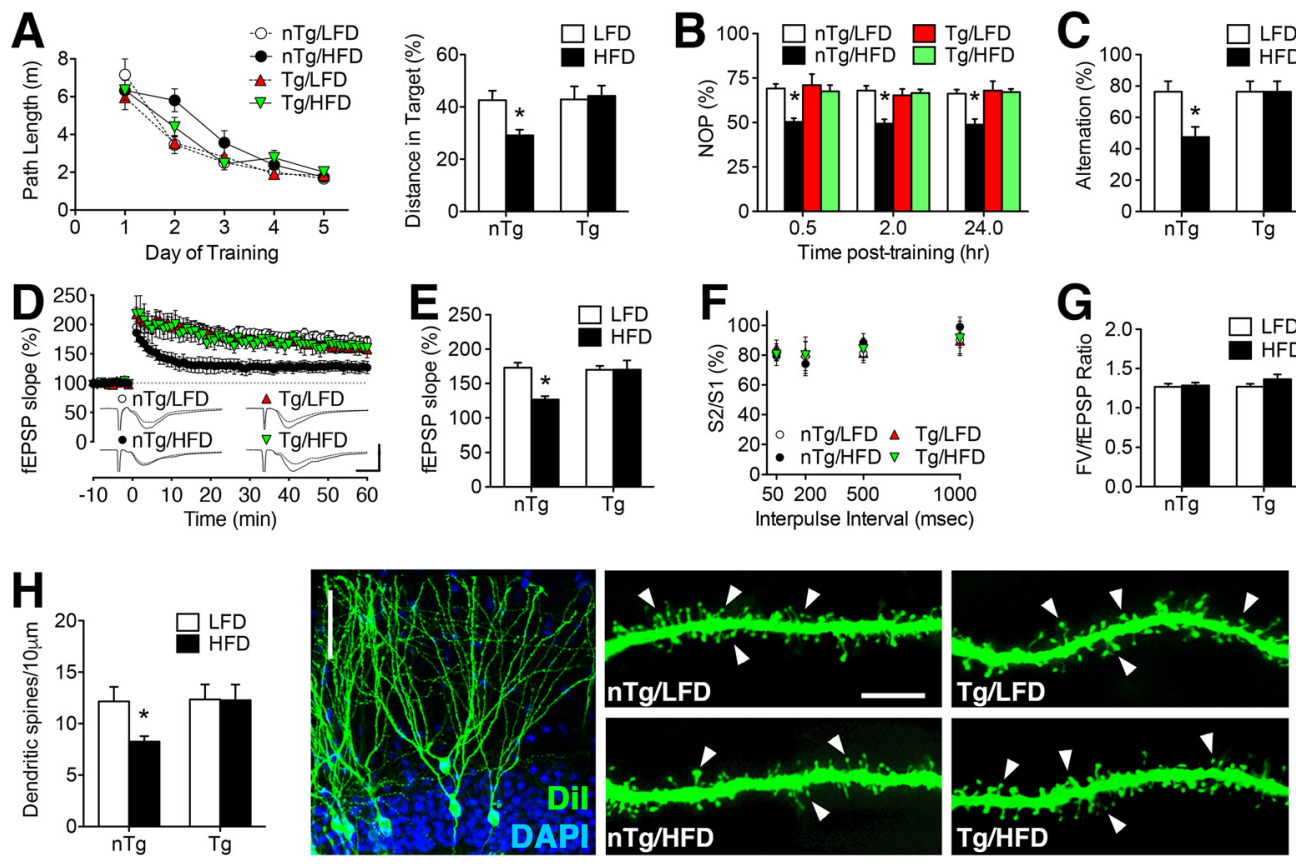

Figure 7. Adora2a-mediated cerebrovascular disruption impairs hippocampus-dependent memory and synaptic plasticity in mice with diet-induced insulin resistance. $A$, Left graph shows path lengths during acquisition trials in Tg and nTg mice maintained on HFD or LFD. Right graph shows proportion of total distance in target quadrant during the probe trial. $\boldsymbol{B}$, Deficits in novel object preference (NOP) in HFD mice were mediated by endothelial Adora2a. C, Endothelial Adora2a ablation maintains spontaneous alternation behavior in the Y-maze after 16 weekHFD. A-C, Bar or symbol height represents the average of $(n=12-16)$ mice per condition. $\boldsymbol{D}$, Impairment of dentate gyrus LTP is attributable to endothelial Adora2a in HFD mice. Representative fEPSPs (inset) show responses during baseline recording and 50 - $60 \mathrm{~min}$ post-tetanic stimulation. For traces, scale bar: (bottom right) $y$-axis, $1.0 \mathrm{mV}$; $x$-axis, $10 \mathrm{~ms}$. E, Percentage increase in field EPSPs (fEPSP) during minutes $50-60$ of recording. $\boldsymbol{F}$, Presynaptic paired-pulse depression was unaffected by diet or genotype. $\mathbf{G}$, The input/output relationship was similar in all groups of mice. $\boldsymbol{D}-\mathbf{G}$, Bar or symbol height represents $(n=8-12)$ slices from $(n=6-8)$ mice per condition. $\boldsymbol{H}$, Dendritic spine density along the secondary and tertiary dendrites of dentate granule neurons was reduced in nTg/HFD mice, but not in Tg/HFD mice. Micrographs (right) show representative images of cells and dendritic segments labeled with the lipophilic membrane tracer Dil with arrowheads indicating dendritic spines. Scale bars: far left, $50 \mu \mathrm{m}$; for images of dendritic segments: (in nTg/LFD) for all other micrographs, $10 \mu \mathrm{m}$. G, Bar height represents the average of ( $n=4-6)$ mice per condition. For all graphs, error bars represent SEM and asterisk indicates significance at $p<0.05$ by ANOVA with Bonferroni's post hoc.

diet $\times$ genotype interaction: $\left.F_{(1,19)}=5.15, p<0.05\right)$, consistent with the postsynaptic pattern of synaptic functional outcomes in the LTP experiments. Together, these changes in synaptic and behavioral plasticity are consistent with a role for Adora2a-mediated BBB breakdown in obesity-induced cognitive impairment.

\section{Discussion}

These studies demonstrate that endothelial Adora2a activation promotes $\mathrm{BBB}$ breakdown during the transition from obesity to diet-induced insulin resistance, and that vascular dysfunction underlies cognitive impairment in mice maintained on an obesogenic diet. Cerebrovascular protection conferred resistance to cognitive impairment and synaptic loss, suggesting that neurocognitive dysfunction in obesity originates from the vasculature. We observed that systemic Adora2a antagonism rescued longterm potentiation in Wt mice on HFD, while direct application of the Adora2a antagonist SCH58261 to hippocampal slices had no effect. When interpreted in the context of behavioral and synaptic rescue following inducible ablation of endothelial Adora2a, the results observed with the antagonist strongly implicate the recovery of BBB integrity as an upstream mechanism for improvements in memory and synaptic plasticity. Adenosine also regulates neuronal synaptic plasticity and cognition (Dias et al., 2013), and there are several potential scenarios in which endothelial Adora2a ablation could impact cells in the CNS parenchyma. Both the high-affinity Adoral and the lower-affinity Adora2a receptor are expressed by dentate granule neurons and CA1 pyramidal neurons (Cunha et al., 1994). Under normal physiological conditions, Adoral activation suppresses LTP, while Adora2a activation supports synaptic plasticity (Dias et al., 2013). In aged rodents, changes in extracellular metabolism of ATP increase activation of the lower-affinity Adora2a by adenosine (Cunha et al., 2001). Age-related changes in ambient adenosine occur in parallel with upregulation of Adora2a expression and binding (Cunha et al., 1995). In mouse models of AD, systemic Adora2a antagonism rescues cognition and CA1 LTP (Silva et al., 2018). Although it is tempting to speculate that restoration of function in $\mathrm{AD}$ mice might be linked with normalization of $\mathrm{BBB}$ integrity, it is also possible that reductions in neuronal Adora2a activation play a role, based on reports that viral vector-mediated downregulation of Adora2a rescued LTP at associational-commissural synapses in hippocampal area CA3 in AD mice (Viana da Silva et al., 2016). Further studies will be required to determine what mechanism, if any, might link vascular Adora2a with possible changes in adenosine signaling among other cell types with dietary obesity.

We and others have previously reported loss of tight junction proteins in rodent models of obesity and diabetes (Kanoski et al., 2010; Ouyang et al., 2014; Stranahan et al., 2016). In the current report, endothelial Adora2a knock-out mice were protected against reductions in the tight junction proteins claudin-5 and occludin with high-fat feeding, and did not exhibit reductions in gene transcription for the tight junction proteins claudin-1 and Jam2. Tg mice were also protected against the upregulation of Adora2a gene expression observed in microvessel preparations from nTg mice on HFD, and the inverse correlation between 
Adora2a upregulation and tight junction gene expression raises questions about potential mechanisms linking these processes. Under normal physiological conditions, activation of Adora2a recruits the canonical $G \alpha$ s protein signaling cascade resulting in cAMP production and CREB-mediated gene transcription. In the presence of inflammatory stimuli, Adora2a activation potentiates NFKb-mediated gene transcription (Tacchini et al., 2008) and in diabetic retinopathy, downregulation of endothelial claudin-5 was shown to be NFKb-dependent (Aveleira et al., 2010). We observed Adora2a-mediated vascular inflammation in the CNS, which could elicit NFKb-mediated downregulation of claudin-1 and claudin-5, as shown in the diabetic retina (Aveleira et al., 2010). It may therefore be possible that obesity-induced upregulation of endothelial Adora2a suppresses tight junction gene expression via NFkb, and future investigations are warranted to examine this potential mechanism.

Pericyte regression coincided with loss of $\mathrm{BBB}$ integrity in $\mathrm{Wt}$ mice maintained on HFD and was eliminated by endothelial Adora2a ablation. Pericytes and other mural cells express Adora2a at higher levels than endothelial cells, based on a recent RNA sequencing study. The study identified substantial transcriptional heterogeneity between endothelial cells in arterioles, venuoles, and capillaries. Pericytes and vascular smooth muscle cells exhibited more consistent transcriptional profiles, including high levels of Adora2a gene expression when examined in aggregate across all regions of the adult mouse brain. Pericytes are typically grouped with endothelial cells as the luminal component of the $\mathrm{BBB}$, but are actually separated from endothelial cells by the pericyte basement membrane (Yao et al., 2014). Given that the Adora2a antagonist SCH58261 crosses BBB (Yang et al., 2007), another potential explanation for normalization of BBB integrity involves direct actions of SCH58261 on pericytes. This interpretation is favored by the higher level of Adora2a expression among pericytes under intact conditions (Vanlandewijck et al., 2018), and also cannot be ruled out in the Adora2a knock-out model, given that pericytes also express the Tek/Tie 2 promoter, albeit at substantially lower levels than endothelial cells (Teichert et al., 2017). However, it is also possible that hippocampal endothelial cells might express Adora2a at higher levels than other brain regions, given the heterogeneity of transcriptional signatures reported in whole-brain endothelial cell preparations. Regional heterogeneity could arise due to cell autonomous differences in gene expression among endothelial cells, or because of the non-uniform distribution of arterioles, venuoles, and capillaries in different areas of the brain (Xiong et al., 2017). Although the relative contributions of endothelial cells and pericytes to obesity-induced BBB breakdown are not fully resolved by the results in this report, it is clear that Adora2a-mediated vascular dysfunction underlies cognitive and synaptic deficits in mice with diet-induced insulin resistance.

We observed duration-dependent disruption of BBB structure and function in mice maintained on an obesogenic HFD. This work complements and extends previous demonstrations of transient increases in BBB permeability with acute (2-3 d) HFD feeding (Jais et al., 2016). These studies identified a role for perivascular macrophages in the resolution of BBB opening after acute HFD consumption (Jais et al., 2016). Specifically, perivascular macrophage-derived VEGF was shown to restore Glut1 expression in brain endothelial cells after 4 weeks of HFD, and this restoration was required for counter-regulation of initial increases in BBB permeability after 1 week of HFD (Jais et al., 2016). The current findings suggest that reductions in microvascular Glut1 re-emerge after longer durations of HFD, when mice begin to exhibit fasting hyperglycemia and insulin resistance. It is possible that recurrent downregulation of microvascular Glut1 may be due to exhaustion of the immunovascular compensation mechanisms identified by Jais et al. (2016). Interrogation of duration-dependent mechanisms for neurovascular disruption and its counter-regulation is challenging and would benefit from the development of novel in vivo imaging strategies for longitudinal studies.

The literature on BBB dysfunction in chronic inflammatory conditions includes many loss-of-function models, and very few models with resistance to BBB opening. Only a few targets have been identified for BBB gain-of-function, and a smaller subset of those targets have been evaluated in models with cell typespecificity. Re-expressing vascular histamine $\mathrm{H} 1$ receptors (H1R) in mice with whole-body deletion of H1R confers resistance to BBB breakdown in experimental autoimmune encephalitis (Lu et al., 2010), but given the diverse roles of H1R in different tissues, it would be ineffective to conduct diet-induced obesity experiments against a background of H1R deletion. Similarly, vascular ablation of the orphan G-protein-coupled receptor GPR124 blocked embryonic angiogenesis in the brain, and because the barrier properties of the cerebral vasculature emerge at the same time during embryonic development, the authors concluded that their model lacked both angiogenic and barrier-forming properties (Kuhnert et al., 2010). These authors also generated mice with endothelial overexpression of GPR124, and although those mice survive to adulthood, more than half exhibit spontaneous arteriovenous malformations, limiting their usefulness for longterm diet studies. To date, only a single promoter exists with relative selectivity for endothelial cells of the nervous system. Solute carrier organic anion transporter $1 \mathrm{C} 1$ (Slo1c1) is primarily expressed in endothelial cells of the brain and spinal cord, but this promoter was also expressed by neurons in the hippocampal dentate gyrus and in sparse populations of cortical astrocytes. Interestingly, inducible ablation of Glut1 in adult Slo1c1 ${ }^{\text {creER }}$ mice resulted in premature lethality, likely due to the impact of impaired glucose entry into the CNS (Jais et al., 2016). Selectivity and global viability are persistent technical hurdles for studying the $\mathrm{BBB}$, but a recent genomics and proteomics study identified several targets with substantially greater enrichment in CNS endothelial cells, relative to peripheral endothelial cells (Zuchero et al., 2016), and novel animal models will likely arise from this research.

Given that the cerebral vasculature is a more accessible target for potential therapies, understanding vascular regulation of cognitive impairment in obesity may prove to be a promising avenue for prevention of obesity-associated neurological risk factors. Additionally, changes in BBB permeability with human obesity could impact the bioavailability of peripherally administered compounds in the nervous system, with the potential for amplification or diminution of cellular effects. These factors, together with unanswered questions about signaling mechanisms for cerebrovascular communication with the CNS, will likely drive future research on neurovascular interactions in obesity and other chronic diseases.

\section{References}

Abbott NJ, Rönnbäck L, Hansson E (2006) Astrocyte-endothelial interactions at the blood-brain barrier. Nat Rev Neurosci 7:41-53.

Aveleira CA, Lin CM, Abcouwer SF, Ambrósio AF, Antonetti DA (2010) TNF- $\alpha$ signals through PKC $\zeta / \mathrm{NF}-\kappa \mathrm{B}$ to alter the tight junction complex and increase retinal endothelial cell permeability. Diabetes 59:2872-2882.

Banks WA (2015) The blood-brain barrier in neuroimmunology: tales of separation and assimilation. Brain Behav Immun 44:1-8. 
Bauer HC, Krizbai IA, Bauer H, Traweger A (2014) "You shall not pass": tight junctions of the blood-brain barrier. Front Neurosci 8:392.

Bell RD, Winkler EA, Sagare AP, Singh I, LaRue B, Deane R, Zlokovic BV (2010) Pericytes control key neurovascular functions and neuronal phenotype in the adult brain and during brain aging. Neuron 68:409-427.

Biessels GJ, Reagan LP (2015) Hippocampal insulin resistance and cognitive dysfunction. Nat Rev Neurosci 16:660-671.

Buckman LB, Thompson MM, Moreno HN, Ellacott KL (2013) Regional astrogliosis in the mouse hypothalamus in response to obesity. J Comp Neurol 521:1322-1333.

Calvo-Ochoa E, Hernández-Ortega K, Ferrera P, Morimoto S, Arias C (2014) Short-term high-fat-and-fructose feeding produces insulin signaling alterations accompanied by neurite and synaptic reduction and astroglial activation in the rat hippocampus. J Cereb Blood Flow Metab 34:1001-1008.

Carman AJ, Mills JH, Krenz A, Kim DG, Bynoe MS (2011) Adenosine receptor signaling modulates permeability of the blood-brain barrier. J Neurosci 31:13272-13280.

Cavalieri B (1635) Geometry, developed by a new method through the indivisibles of the continua. Bononiae: Typis Clementis Ferronij.

Cunha RA, Johansson B, van der Ploeg I, Sebastião AM, Ribeiro JA, Fredholm BB (1994) Evidence for functionally important adenosine A2a receptors in the rat hippocampus. Brain Res 649:208-216.

Cunha RA, Constantino MC, Sebastião AM, Ribeiro JA (1995) Modification of $\mathrm{A} 1$ and $\mathrm{A} 2 \mathrm{a}$ adenosine receptor binding in aged striatum, hippocampus and cortex of the rat. Neuroreport 6:1583-1588.

Cunha RA, Almeida T, Ribeiro JA (2001) Parallel modification of adenosine extracellular metabolism and modulatory action in the hippocampus of aged rats. J Neurochem 76:372-382.

Daneman R, Prat A (2015) The blood-brain barrier. Cold Spring Harb Perspect Biol 7:a020412.

Dey A, Hao S, Erion JR, Wosiski-Kuhn M, Stranahan AM (2014) Glucocorticoid sensitization of microglia in a genetic mouse model of obesity and diabetes. J Neuroimmunol 269:20-27.

Dias RB, Rombo DM, Ribeiro JA, Henley JM, Sebastião AM (2013) Adenosine: setting the stage for plasticity. Trends Neurosci 36:248-257.

Elias MF, Elias PK, Sullivan LM, Wolf PA, D’Agostino RB (2005) Obesity, diabetes and cognitive deficit: the framingham heart study. Neurobiol Aging 26:11-16.

Erion JR, Wosiski-Kuhn M, Dey A, Hao S, Davis CL, Pollock NK, Stranahan AM (2014) Obesity elicits interleukin 1-mediated deficits in hippocampal synaptic plasticity. J Neurosci 34:2618-2631.

Fredholm BB, Lindström K, Dionisotti S, Ongini E (1998) [ $\left.{ }^{3} \mathrm{H}\right] \mathrm{SCH} 58261$, a selective adenosine A2A receptor antagonist, is a useful ligand in autoradiographic studies. J Neurochem 70:1210-1216.

Gustafson DR, Karlsson C, Skoog I, Rosengren L, Lissner L, Blennow K (2007) Mid-life adiposity factors relate to blood-brain barrier integrity in late life. J Intern Med 262:643-650.

Hao S, Dey A, Yu X, Stranahan AM (2016) Dietary obesity reversibly induces synaptic stripping by microglia and impairs hippocampal plasticity. Brain Behav Immun 51:230-239.

Ho AJ, Raji CA, Becker JT, Lopez OL, Kuller LH, Hua X, Lee S, Hibar D, Dinov ID, Stein JL, Jack CR Jr, Weiner MW, Toga AW, Thompson PM (2010) Obesity is linked with lower brain volume in $700 \mathrm{AD}$ and MCI patients. Neurobiol Aging 31:1326-1339.

Isaacs KR, Anderson BJ, Alcantara AA, Black JE, Greenough WT (1992) Exercise and the brain: angiogenesis in the adult rat cerebellum after vigorous physical activity and motor skill learning. J Cereb Blood Flow Metab 12:110-119.

Jais A, Solas M, Backes H, Chaurasia B, Kleinridders A, Theurich S, Mauer J, Steculorum SM, Hampel B, Goldau J, Alber J, Förster CY, Eming SA, Schwaninger M, Ferrara N, Karsenty G, Brüning JC (2016) Myeloidcell-derived VEGF maintains brain glucose uptake and limits cognitive impairment in obesity. Cell 165:882-895.

Kanneganti TD, Dixit VD (2012) Immunological complications of obesity. Nat Immunol 13:707-712.

Kanoski SE, Zhang Y, Zheng W, Davidson TL (2010) The effects of a highenergy diet on hippocampal function and blood-brain barrier integrity in the rat. J Alzheimers Dis 21:207-219.

Koni PA, Joshi SK, Temann UA, Olson D, Burkly L, Flavell RA (2001) Conditional vascular cell adhesion molecule 1 deletion in mice: impaired lymphocyte migration to bone marrow. J Exp Med 193:741-754.
Kuhnert F, Mancuso MR, Shamloo A, Wang HT, Choksi V, Florek M, Su H, Fruttiger M, Young WL, Heilshorn SC, Kuo CJ (2010) Essential regulation of CNS angiogenesis by the orphan G protein-coupled receptor GPR124. Science 330:985-999.

Lu C, Diehl SA, Noubade R, Ledoux J, Nelson MT, Spach K, Zachary JF, Blankenhorn EP, Teuscher C (2010) Endothelial histamine H1 receptor signaling reduces blood-brain barrier permeability and susceptibility to autoimmune encephalomyelitis. Proc Natl Acad Sci U S A 107:1896718972.

Mills JH, Thompson LF, Mueller C, Waickman AT, Jalkanen S, Niemela J, Airas L, Bynoe MS (2008) CD73 is required for efficient entry of lymphocytes into the central nervous system during experimental autoimmune encephalomyelitis. Proc Natl Acad Sci U S A 105:9325-9330.

Miner JJ, Daniels BP, Shrestha B, Proenca-Modena JL, Lew ED, Lazear HM, Gorman MJ, Lemke G, Klein RS, Diamond MS (2015) The TAM receptor mertk protects against neuroinvasive viral infection by maintaining blood-brain barrier integrity. Nat Med 21:1464-14672.

Montagne A, Barnes SR, Sweeney MD, Halliday MR, Sagare AP, Zhao Z, Toga AW, Jacobs RE, Liu CY, Amezcua L, Harrington MG, Chui HC, Law M, Zlokovic BV (2015) Blood-brain barrier breakdown in the aging human hippocampus. Neuron 85:296-302.

Ouyang S, Hsuchou H, Kastin AJ, Wang Y, Yu C, Pan W (2014) Dietinduced obesity suppresses expression of many proteins at the bloodbrain barrier. J Cereb Blood Flow Metab 34:43-51.

Silva AC, Lemos C, Gonçalves FQ, Pliássova AV, Machado NJ, Silva HB, Canas PM, Cunha RA, Lopes JP, Agostinho P (2018) Blockade of adenosine $\mathrm{A} 2 \mathrm{~A}$ receptors recovers early deficits of memory and plasticity in the triple transgenic mouse model of Alzheimer's disease. Neurobiol Dis 117: $72-81$.

Stranahan AM, Norman ED, Lee K, Cutler RG, Telljohann RS, Egan JM, Mattson MP (2008) Diet-induced insulin resistance impairs hippocampal synaptic plasticity and cognition in middle-aged rats. Hippocampus 18:1085-1088.

Stranahan AM, Lee K, Becker KG, Zhang Y, Maudsley S, Martin B, Cutler RG, Mattson MP (2010) Hippocampal gene expression patterns underlying the enhancement of memory by running in aged mice. Neurobiol Aging 31:1937-1949.

Stranahan AM, Haberman RP, Gallagher M (2011) Cognitive decline is associated with reduced reelin expression in the entorhinal cortex of aged rats. Cereb Cortex 21:392-400.

Stranahan AM, Hao S, Dey A, Yu X, Baban B (2016) Blood-brain barrier breakdown promotes macrophage infiltration and cognitive impairment in leptin receptor-deficient mice. J Cereb Blood Flow Metab 36:21082121.

Tacchini L, Gammella E, De Ponti C, Recalcati S, Cairo G (2008) Role of HIF- 1 and NF- $\kappa$ B transcription factors in the modulation of transferrin receptor by inflammatory and anti-inflammatory signals. J Biol Chem 283:20674-20686.

Teichert M, Milde L, Holm A, Stanicek L, Gengenbacher N, Savant S, Ruckdeschel T, Hasanov Z, Srivastava K, Hu J, Hertel S, Bartol A, Schlereth K, Augustin HG (2017) Pericyte-expressed Tie2 controls angiogenesis and vessel maturation. Nat Commun 8:16106.

Vanlandewijck M, He L, Mäe MA, Andrae J, Ando K, Del Gaudio F, Nahar K, Lebouvier T, Laviña B, Gouveia L, Sun Y, Raschperger E, Räsänen M, Zarb Y, Mochizuki N, Keller A, Lendahl U, Betsholtz C (2018) A molecular atlas of cell types and zonation in the brain vasculature. Nature 554:475480.

van Praag H, Lucero MJ, Yeo GW, Stecker K, Heivand N, Zhao C, Yip E, Afanador M, Schroeter H, Hammerstone J, Gage FH (2007) Plantderived flavanol ( - epicatechin enhances angiogenesis and retention of spatial memory in mice. J Neurosci 27:5869-5878.

Viana da Silva S, Haberl MG, Zhang P, Bethge P, Lemos C, Gonçalves N, Gorlewicz A, Malezieux M, Gonçalves FQ, Grosjean N, Blanchet C, Frick A, Nägerl UV, Cunha RA, Mulle C (2016) Early synaptic deficits in the APP/PS1 mouse model of Alzheimer's disease involve neuronal adenosine A2A receptors. Nat Commun 7:11915.

Wang HL, Lai TW (2014) Optimization of Evans blue quantitation in limited rat tissue samples. Sci Rep 4:6588.

Wang T, Town T, Alexopoulou L, Anderson JF, Fikrig E, Flavell RA (2004) Toll-like receptor 3 mediates west nile virus entry into the brain causing lethal encephalitis. Nat Med 10:1366-1373. 
Ward MA, Carlsson CM, Trivedi MA, Sager MA, Johnson SC (2005) The effect of body mass index on global brain volume in middle-aged adults: a cross sectional study. BMC Neurol 5:23.

Whitmer RA, Gustafson DR, Barrett-Connor E, Haan MN, Gunderson EP, Yaffe K (2008) Central obesity and increased risk of dementia more than three decades later. Neurology 71:1057-1064.

Wosiski-Kuhn M, Erion JR, Gomez-Sanchez EP, Gomez-Sanchez CE, Stranahan AM (2014) Glucocorticoid receptor activation impairs hippocampal plasticity by suppressing BDNF expression in obese mice. Psychoneuroendocrinology 42:165-177.

Wu Z, Hofman FM, Zlokovic BV (2003) A simple method for isolation and characterization of mouse brain microvascular endothelial cells. J Neurosci Methods 130:53-63.

Xiong B, Li A, Lou Y, Chen S, Long B, Peng J, Yang Z, Xu T, Yang X, Li X, Jiang T, Luo Q, Gong H (2017) Precise cerebral vascular atlas in stereotaxic coordinates of whole mouse brain. Front Neuroanat 11:128.

Xu W, Caracciolo B, Wang HX, Winblad B, Bäckman L, Qiu C, Fratiglioni L (2010) Accelerated progression from mild cognitive impairment to dementia in people with diabetes. Diabetes 59:2928-2935.
Yang M, Soohoo D, Soelaiman S, Kalla R, Zablocki J, Chu N, Leung K, Yao L, Diamond I, Belardinelli L, Shryock JC (2007) Characterization of the potency, selectivity, and pharmacokinetic profile for six adenosine A2A receptor antagonists. Naunyn Schmiedebergs Arch Pharmacol 375:133144.

Yao Y, Chen ZL, Norris EH, Strickland S (2014) Astrocytic laminin regulates pericyte differentiation and maintains blood brain barrier integrity. Nat Commun 5:3413.

Yi CX, Gericke M, Krüger M, Alkemade A, Kabra DG, Hanske S, Filosa J, Pfluger P, Bingham N, Woods SC, Herman J, Kalsbeek A, Baumann M, Lang R, Stern JE, Bechmann I, Tschöp MH (2012) High calorie diet triggers hypothalamic angiopathy. Mol Metab 1:95-100.

Zhao Z, Nelson AR, Betsholtz C, Zlokovic BV (2015) Establishment and dysfunction of the blood-brain barrier. Cell 163:1064-1078.

Zuchero YJ, Chen X, Bien-Ly N, Bumbaca D, Tong RK, Gao X, Zhang S, Hoyte K, Luk W, Huntley MA, Phu L, Tan C, Kallop D, Weimer RM, Lu Y, Kirkpatrick DS, Ernst JA, Chih B, Dennis MS, Watts RJ (2016) Discovery of novel blood-brain barrier targets to enhance brain uptake of therapeutic antibodies. Neuron 89:70-82. 